\title{
Populations of Stellar-mass Black Holes from Binary Systems
}

\author{
Grzegorz Wiktorowicz $^{1,2,3}$, Łukasz Wyrzykowski ${ }^{3}$ (D) Martyna Chruslinska ${ }^{4}$, Jakub Klencki ${ }^{4}$, Krzysztof A. Rybicki ${ }^{3}$, and \\ Krzysztof Belczynski ${ }^{5}$ \\ ${ }^{1}$ National Astronomical Observatories, Chinese Academy of Sciences, Beijing 100101, People's Republic of China; gwiktoro@astrouw.edu.pl \\ ${ }^{2}$ School of Astronomy \& Space Science, University of the Chinese Academy of Sciences, Beijing 100012, People's Republic of China \\ ${ }^{3}$ Astronomical Observatory, Warsaw University, Al. Ujazdowskie 4, 00-478 Warsaw, Poland \\ ${ }^{4}$ Institute of Mathematics, Astrophysics and Particle Physics, Radboud University Nijmegen, P.O. Box 9010, 6500 GL, The Netherlands \\ ${ }^{5}$ Nicolaus Copernicus Astronomical Center, Polish Academy of Sciences, Bartycka 18, 00-716 Warsaw, Poland \\ Received 2019 July 25; revised 2019 September 13; accepted 2019 September 17; published 2019 October 24
}

\begin{abstract}
In large and complicated stellar systems like galaxies, it is difficult to predict the number and characteristics of a black hole (BH) population. Such populations may be modeled as an aggregation of homogeneous (i.e., having uniform star formation history and the same initial chemical composition) stellar populations. Using realistic evolutionary models, we predict the abundances and properties of $\mathrm{BHs}$ formed from binaries in these environments. We show that the BH population will be dominated by single BHs originating from binary disruptions and stellar mergers. Furthermore, we discuss how BH populations are influenced by such factors as initial parameters, metallicity, initial mass function, and natal kick models. As an example application of our results, we estimate that about 26 microlensing events happen every year in the direction of the Galactic Bulge due to BHs in a survey like OGLE-IV. Our results may be used to perform in-depth studies related to realistic BH populations, such as observational predictions for space survey missions like Gaia or Einstein Probe. We prepared a publicly available database with the raw data from our simulations to be used for more in-depth studies.
\end{abstract}

Unified Astronomy Thesaurus concepts: Black holes (162); Astrophysical black holes (98); Stellar populations (1622); Binary stars (154); X-ray binary stars (1811); Compact objects (288); Gravitational microlensing (672); Gravitational waves (678); Stellar evolution (1599)

\section{Introduction}

A black hole $(\mathrm{BH})$ is defined as a region in space from which nothing, even light, can escape (for a recent review, see Bambi 2018). BHs may be detected when interacting with other objects (e.g., in X-ray binaries, XRB; Remillard \& McClintock 2006; Corral-Santana et al. 2016), as gravitational wave sources (e.g., Abbott et al. 2016a, 2017a), when they interfere with radiation (e.g., as gravitational lenses; Wyrzykowski et al. 2016), or in noninteracting binaries by observations of their companions (e.g., Mashian \& Loeb 2017; Thompson et al. 2018). It was also proposed that single $\mathrm{BHs}$ (SBHs) may be observed as X-ray novae (e.g., Matsumoto et al. 2018).

The so-called stellar-mass BHs, that is, stellar-origin BHs and $\mathrm{BHs}$ originating from mergers of stars or stellar-origin compact objects, with masses from $\sim 5$ to possibly a few $\times 100 M_{\odot}$, are final stages of massive star evolution (e.g., Neugebauer 2003) and are distinguished from other subgroups such as supermassive BHs $\left(M_{\mathrm{BH}} \gtrsim 10^{6} M_{\odot}\right.$; e.g., Ferrarese \& Ford 2005), intermediate-mass BHs $\left(\sim 10^{2}<M_{\mathrm{BH}}<\sim 10^{5} M_{\odot}\right.$; e.g., Mezcua 2017; Koliopanos 2017), and hypothetical primordial BHs (e.g., Chapline 1975; Khlopov 2010). Hereafter, we focus exclusively on stellar-mass BHs.

Corral-Santana et al. (2016) provided a list of 59 BHs in transient XRBs. Twenty-two of them have dynamically confirmed mass estimates (see Casares et al. 2017 for a recent list). Five BHs were detected in high-mass XRBs (defined as having a donor mass above $10 M_{\odot}$ ). Recently, gravitational waves made it possible to discover first double $\mathrm{BH}$ merger events (Abbott et al. 2016a, 2016b, 2017a, 2017b) and a double neutron star (NS) merger GW170817, which may have formed a low-mass BH (Pooley et al. 2018). Interestingly, Adams et al. (2017) observed a $\sim 25 M_{\odot}$ star to disappear after a short brightening, which may be interpreted as a $\mathrm{BH}$-formation event. Up to now, no SBHs were confirmed (e.g., Tsuna et al. 2018).

Previous studies of $\mathrm{BH}$ populations are usually outdated and do not take into account recent progress in our understanding of massive star evolution (e.g., Langer 2012; Vink et al. 2015; Vink 2015). The earliest estimations predicted $\sim 100$ million BHs in the Milky Way (MW) galaxy (Shapiro \& Teukolsky 1983 ) with as much as $45 \%$ of supernovae occurring in close binaries (Tutukov et al. 1992). Timmes et al. (1996) provided an upper limit of $1.4 \times 10^{9}$, and Samland (1998) estimated $1.8 \times 10^{8}$ BHs in the galaxy, taking into account changes in star formation rate (SFR). Belczynski et al. (2002a, 2004) used the StarTrack population synthesis code in its earlier version to investigate $\mathrm{BHs}$ formed in star formation (SF) bursts. Their results indicate that most $\mathrm{BHs}$ in the MW are actually single objects (not in binaries), whereas those that remain bound will have mostly main-sequence (MS) companions, provided that the stellar population is not very old.

Recent studies do not provide estimations for parameter distributions of the entire $\mathrm{BH}$ population. For example, Elbert et al. (2018) predicted $\sim 10^{8} \mathrm{BHs}$ in a MW-type galaxy, but their approach does not take into account binary interactions directly and focuses on binary $\mathrm{BHs}(\mathrm{BH}+\mathrm{BH})$ only. Lamberts et al. (2018) performed a detailed cosmological simulation of the MW evolution (including, e.g., changes in metallicity) and predicted that $\sim 1$ million $\mathrm{BH}$ binaries have already merged in the galaxy, whereas $\sim 3$ million are still present. However, their study does not account for BHs with lower-mass companions or disrupted systems, which may actually constitute the bulk of the $\mathrm{BH}$ population (Belczynski et al. 2004). 
Although the current population of $\mathrm{BHs}$ is predicted to be mainly single, their progenitors did not have to be single stars. Due to a natal kick (NK; e.g., Lyne \& Lorimer 1994; Janka 2013), a binary (especially with large orbital separation or a low-mass companion) may be disrupted (Iben \& Tutukov 1997). Recent observational studies (see, e.g., Sana 2017 for a review) suggest that even $\gtrsim 90 \%$ of massive stars (BH progenitors) are born in binaries (or multiple systems). The presence of a companion may significantly affect the evolution of a progenitor and, therefore, final properties of a BH. Some studies used a simplified approach. For example, Elbert et al. (2018) encapsulated all of the interactions in just two parameters. In this study, we focused on a detailed consideration of binary interactions, but we neglected any higher-order systems (e.g., Toonen et al. 2016).

A serious problem in obtaining estimations of $\mathrm{BH}$ populations is the lack of knowledge about the stellar environment. Taking the MW as an example, not even the total stellar mass is well constrained, let alone the star formation history (SFH) or the distribution and motions of stars. What is more, a galaxy with a complicated evolution and structure cannot be modeled with a uniform population of objects evolved from a single SF burst. Therefore, in our approach, we take into account a range of evolutionary models with different initial parameters. Such results can be joined together according to the SFH and chemical evolution of a stellar population in order to obtain more realistic distributions.

Specifically, we propose a step-by-step approach in which we use results from modeling of homogeneous and scalable $\mathrm{BH}$ populations in order to build complicated (nonhomogeneous) stellar systems like galaxies. We provide the estimates for a simplified MW galaxy as an example (for detailed modeling of the MW galaxy, see Olejak et al. 2019). However, our results may be used to simulate virtually any large stellar population providing the dynamical interactions between stars may be neglected (it is not true, e.g., for globular clusters).

The two most important factors that influence $\mathrm{BH}$ populations are the metallicity and NKs. The metallicity of a star significantly affects the mass lost in stellar wind and, therefore, the mass of the final compact object (Fryer \& Kalogera 2001). Belczynski et al. (2010a) showed that the maximal BH mass for a solar-metallicity environment is $\sim 15 M_{\odot}$, whereas for lower metallicity environments it may reach $\sim 80 M_{\odot}$. However, their study did not consider the pair-instability supernova and pairinstability pulsation supernova (e.g., Woosley 2017, 2019) and was performed for single stars only. Both pair-instability supernovae and pair-instability pulsation supernova are believed to produce the second "mass gap" in the distribution of $\mathrm{BH}$ masses between $\sim 50$ and $135 M_{\odot}$ (Belczynski et al. 2016a; Spera \& Mapelli 2017; Marchant et al. 2019; Woosley 2019). However, in binary systems, the masses of BHs can fill this range because of mass-transfer (MT) phases and mergers (e.g., Spera et al. 2019).

Uncertainties in the modeling of the common envelope (CE) phase add additional significant error to the predictions of binary evolution (for a review, see Ivanova et al. 2013). The CE seems to be essential for the formation of XRBs where a strong reduction of initial separation is necessary to produce a Roche lobe overflow (RLOF) or significant accretion from wind. However, it was shown (Wiktorowicz et al. 2014) that different prescriptions for the CE phase give similar results with respect to the population of XRBs. In contrast, the formation of close double compact objects (DCOs), with time to merger smaller than the Hubble time, which also typically involves the CE phase, is highly influenced by the adopted CE model (e.g., Dominik et al. 2012). Nonetheless, as we show in this paper, only a small part of the total $\mathrm{BH}$ population resides in XRBs and close DCOs. Most stellar mergers of isolated binaries occur as a result of failed CE ejection, so the CE model may strongly influence the population of BHs originating from merger products. However, even though outcomes of mergers may constitute a significant fraction of a $\mathrm{BH}$ population, the poorly understood physics of stellar mergers and postmerger evolution make it impossible to reliably describe the population of these objects. In this paper, therefore, we do not include the analysis of CE models.

The fraction of binaries that remain bound after a supernova (SN) explosion as well as peculiar velocities of BHs are particularly sensitive to the assumptions about the NKs. The former affects the predicted ratio of the number of SBHs to those found in binaries. The latter influences the spatial distribution of BHs in stellar systems (e.g., galaxies). Although the SN mechanism is not well understood, NKs are usually connected to asymmetries arising in this process. The main candidates are mass ejection (e.g., Wongwathanarat et al. 2013), gravitational waves (Bonnell \& Pringle 1995), and neutrinos (e.g., Fryer \& Kusenko 2006). Miller-Jones (2014) derived peculiar velocities of several BH binaries (BHB, i.e., binaries composed of a $\mathrm{BH}$ and a noncompact companion), obtaining values between 19 and $144 \mathrm{~km} \mathrm{~s}^{-1}$. However, it is usually difficult to assess NKs from present-day motions (Fragos et al. 2009; Repetto et al. 2012; Repetto \& Nelemans 2015). Recent analysis performed by Repetto et al. (2017) has shown that at least some of the BHs in the MW should have obtained high NKs $\left(\sim 100 \mathrm{~km} \mathrm{~s}^{-1}\right)$, comparable to those of NSs. On the other hand, Mandel (2016) showed that velocities higher than $\sim 80 \mathrm{~km} \mathrm{~s}^{-1}$ are not necessary, although they cannot be ruled out. Additionally, Jonker \& Nelemans (2004) showed that XRBs with BH accretors have a spatial distribution similar to that of NS accretors, which suggests a similar NK magnitude at birth. See Belczynski et al. (2016c) and references therein for a recent discussion on the BH NKs.

In this paper, we performed the first simulation of the entire $\mathrm{BH}$ population for different evolutionary models. Our analysis is focused on SBHs from binary disruption events (Section 3.1), BHBs, DCOs (Section 3.2), and potential BHs originating from stellar mergers (Section 3.3). We pay particular attention to the description of the results for the standard model (STD), which may depict the MW disk field population, and differences in relation to other models.

The main goals of this work are (1) to describe the general characteristics of the $\mathrm{BH}$ population and its dependence on the most important model parameters (e.g., metallicity, NKs) and (2) to provide a reference point for forthcoming in-depth studies focusing on astrophysical problems involving $\mathrm{BH}$ populations like XRBs, microlensing, and gravitational wave sources, to name a few. Especially educational are possible comparison studies with the results of actual and future surveys focused on gravitational wave sources (e.g., aLIGO, Einstein Telescope, LISA), X-ray sources (e.g., XMM-Newton, Chandra, NuSTAR), or gravitational microlensing (OGLE, Gaia, LSST), which may result in obtaining better constraints for the evolutionary models. 
Table 1

Summary of Models

\begin{tabular}{ll}
\hline \hline Model & \multicolumn{1}{c}{ Difference with Respect to Standard Model } \\
\hline STD & Standard (reference) model: \\
& Solar metallicity $Z_{\odot}$ \\
& Distribution of initial periods \\
& $P(\log P) \sim(\log P)^{-0.55}$ \\
& Distribution of initial eccentricities \\
& $P(e) \sim e^{-0.42}$ \\
& BH/NS natal kicks are drawn from \\
& Maxwellian distribution with $\sigma=265 \mathrm{~km} \mathrm{~s}^{-1}$ \\
& BH natal kicks reduced by fallback \\
& Moderate slope for high-mass end of the IMF \\
& $\Gamma=-2.3$ \\
& Metallicity equal to $10 \% Z_{\odot}$ \\
mid- $Z$ & Metallicity equal to $1 \% Z_{\odot}$ \\
low- $Z$ & Distribution of initial separations log $(a) \sim 1$ \\
SS0 & Distribution of initial eccentricities $P(e) \sim e$ \\
& BH NKs are inversely proportional to the BH's mass \\
NK ${ }_{\mathrm{R}}$ & (Equation $(2))$ \\
& BH/NS natal kick proportional to \\
NK & ratio of ejecta mass and remnant mass $($ Equation $(3))$ \\
& Flat slope for high-mass end of the IMF $(\Gamma=-1.9)$ \\
flat IMF & Steep slope for high-mass end of the IMF $(\Gamma=-2.7)$ \\
steep IMF &
\end{tabular}

Note. All main parameters are provided only for the STD model. For other models, only differences from the STD model are given explicitly.

Particularly, in Section 4 we estimate a number of microlensing events toward the Galactic Bulge. The microlensing method seems to be a promising way to search for SBHs, which, as we show in this work, constitute a vast majority of all BHs in stellar populations.

In order to allow for easier and more flexible use of the data introduced by this paper, we prepared a publicly available database where all of the results are available for download: https://universeathome.pl/universe/bhdb.php.

\section{Methodology}

We utilize a recent version of the StarTrack population synthesis code (Belczynski et al. 2002b, 2008, with further updates). The code has been frequently used to study BHs in XRBs and DCOs (e.g., Dominik et al. 2012, 2013, 2015; Wiktorowicz et al. 2014; Belczynski et al. 2016a; Klencki et al. 2017). Recent updates include, but are not limited to, new prescriptions for wind mass loss from massive stars (Vink 2011), pair-instability supernovae, and pair-instability pulsation supernovae (Belczynski et al. 2016b; Woosley 2017).

Here, we analyze eight main models (Table 1) which differ in parameters that significantly affect the resulting $\mathrm{BH}$ population. Additionally, in the web database, we provide a grid of all 54 models, which allows us to investigate combinations of the main models. For each model, we have simulated the evolution of $2 \times 10^{6}$ binaries from zero-age main-sequence (ZAMS) to disruption, merger, or reaching the age of 15 Gyr. For mergers that are not DCOs, we apply a simple formalism to estimate the endpoint of postmerger single-star evolution (see Section 2.2).

Initial primary masses $\left(M_{\mathrm{a}}\right)$ are drawn from a broken powerlaw distribution (Kroupa et al. 1993) with $\Gamma=-1.3$ for $M_{\mathrm{a}}<$ $0.5 M_{\odot}$ and $\Gamma=-2.2$ for $0.5<M_{\mathrm{a}}<1.0 M_{\odot}$. For initial masses $M_{\mathrm{a}}>1.0 M_{\odot}$, we chose $\Gamma=-2.3$ (STD; Kroupa 2001),
-2.7 (steep initial mass function (IMF) model; Kroupa \& Weidner 2003), or -1.9 (flat IMF model; Schneider et al. 2018). The conclusions of Schneider et al. (2018) were recently revised by Farr \& Mandel (2018), who argued in favor of a much steeper $\Gamma \approx-2.11$ or -2.15 . Being aware of this, we leave the original value in order to emphasize the influence of IMF steepness on the population of BHs. The mass ratio $q=M_{\mathrm{b}} / M_{\mathrm{a}}$ (where $M_{\mathrm{b}}$ is the mass of the secondary, initially less massive star) is drawn from a uniform distribution between $0.08 M_{\odot} / M_{\mathrm{a}}$ and 1 . In the STD model, we assume that the initial distribution of periods is $P(\log P) \sim(\log P)^{-0.55}$ and the distribution of eccentricities is $P(e) \sim e^{-0.42}$ (Sana et al. 2012). Even though the results of Sana et al. (2012) are for stars with initial masses between 15 and $60 M_{\odot}$, we extrapolate them to the entire investigated range $\left(0.08-150 M_{\odot}\right)$. Additionally, in model SS0, we test a distribution of initial separations that is flat in logarithm (Abt 1983) with a maximal value set as $10^{5} R_{\odot}$ and a thermal distribution of eccentricities $(P(e) \sim e$; Duquennoy \& Mayor 1991).

The effects of pair-instability supernovae and pair-instability pulsation supernovae (Woosley 2017) may significantly alter the final BH mass (e.g., Belczynski et al. 2016b). The instability leads to a significant mass loss for massive stars (Woosley et al. 2007) or disruption of the entire star (Heger \& Woosley 2002). Following Belczynski et al. (2016b), we assume that for stars that form massive helium cores $\left(M_{\mathrm{He}}=\right.$ 45-65 $M_{\odot}$ ), all of the envelope above the inner $45 M_{\odot}$ is lost to pulsations. Furthermore, we assume that stars that form heavier helium cores $\left(M_{\mathrm{He}}=65-135 M_{\odot}\right)$ are subject to the pairinstability supernovae and leave no remnant.

The CE phase is important for the evolution of many binaries and may lead to the formation of a much closer system or a merger. In a general situation, we utilize the simple energy balance (Webbink 1984). However, the donor type influences significantly the binary survival during this phase (e.g., Belczynski et al. 2007). MS and Hertzsprung gap (HG) donors lack a clear core-envelope structure (no clear entropy jump; e.g., Taam \& Sandquist 2000; Ivanova \& Taam 2004), so the orbital energy is transferred to the entire star, instead of being transferred to the envelope only, which prevents envelop ejection and leads to a merger. It is not clear when the coreenvelope boundary emerges (late HG or red giant phase), so two models for the treatment of HG donors in CE were introduced (e.g., Dominik et al. 2012). The first one (model B) assumes that a CE phase with an $\mathrm{HG}$ donor always results in a merger. The second one (model A), producing significantly higher DCO merger rates (e.g., Belczynski et al. 2007; Dominik et al. 2012), allows for survival in such situations. We adopt model $\mathrm{B}$ in the present study and analyze the influence of model A in Section 5.4.

We test models with three different metallicities. Most of the models have solar metallicity $\left(Z=0.02=Z_{\odot}\right.$, where $Z_{\odot}$ is the solar metallicity; Villante et al. 2014). We also introduce two models with lower metallicity: the mid- $Z$ model $\left(Z=0.002=10 \% Z_{\odot}\right)$ and the low- $Z$ model $(Z=$ $\left.0.0002=1 \% Z_{\odot}\right)$. Additionally, three different NK models are included in our simulations. Those models are described below.

\subsection{Natal Kick Models}

Hobbs et al. (2005) performed a study of 233 galactic pulsar proper motions and found that for young (i.e., with characteristic age $\left.\tau_{\mathrm{c}}=P / 2 \dot{P}<3 \mathrm{yr}\right)$ pulsars the velocity 
distribution is well fitted with a Maxwellian distribution with $\sigma=265 \mathrm{~km} \mathrm{~s}^{-1}$. Velocities of such a population may quite well resemble the NKs of NSs. However, it is not obvious if the NKs of BHs follow the same distribution. Especially, if NKs are driven by the asymmetries in ejecta, the post-SN fallback may significantly lower the BH's velocity. Proper motion measurements are available only for a few BHs residing in XRBs (Miller-Jones 2014), and the connection between the current peculiar velocity and the NK is not straightforward (e.g., Fragos et al. 2009). On the other hand, the analysis of the positions of XRBs harboring BHs may suggest that distributions of NKs of BHs and NSs are similar (e.g., Jonker \& Nelemans 2004; Repetto et al. 2017; however, see Mandel 2016).

In the standard model, we lower the remnant's NK proportionally to the fallback of material after an SN explosion. Precisely, the NK for a compact object is

$$
V_{\text {kick }}=V_{\text {kick,Maxwell }}\left(1-f_{\mathrm{FB}}\right) \text {, }
$$

where $V_{\text {kick,Maxwell }}$ has a Maxwellian distribution with $\sigma=265 \mathrm{~km} \mathrm{~s}^{-1}$ and $f_{\mathrm{FB}}$ indicates what fraction of ejected mass falls back onto a compact object (see Fryer et al. 2012). This model produces both low-velocity and high-velocity BHs, which is in agreement with observational estimates for XRBs (e.g., Belczynski et al. 2016c).

If NKs are driven by a neutrino-based mechanism, it is predicted that the imposed momentum will be the same for BHs and NSs (e.g., Janka 2013; Rodriguez et al. 2016). Such NKs will be inversely proportional to the mass of a $\mathrm{BH}$ $\left(V_{\text {kick }} \approx M_{\mathrm{BH}}^{-1}\right)$. In the $\mathrm{NK}_{\mathrm{R}}$ model, we assume that the NS NK distribution has a Maxwellian shape with $\sigma=265 \mathrm{~km} \mathrm{~s}^{-1}$, but the $\mathrm{BH}$ NK distribution is lower. Precisely,

$$
\begin{aligned}
& V_{\text {kick,NS }}=V_{\text {kick,Maxwell }}, \\
& V_{\text {kick,BH }}=V_{\text {kick,Maxwell }} \frac{M_{\text {max,NS }}}{M_{\mathrm{BH}}},
\end{aligned}
$$

where $M_{\text {max,NS }}=2.5 M_{\odot}$ is an adopted limit for an NS mass above which it collapses to a $\mathrm{BH}$. It must be noted that, even for low-mass BHs $\left(\sim 5-7 M_{\odot}\right)$, such a prescription will produce significantly lower NKs than the NKs of NSs, which stands in contradiction to the results of, for example, Repetto et al. (2017). Nevertheless, we included this model as a parameter study.

Finally, we incorporated a simple model proposed by Bray \& Eldridge (2018), who suggested that the NK distribution can be described as a linear function of a ratio between the mass of the ejecta $\left(M_{\text {ejecta }}\right)$ and the mass of the remnant $\left(M_{\text {remnant }}\right)$. We applied (model $\mathrm{NK}_{\mathrm{BE}}$ ) their fit to the observations provided by Hobbs et al. (2005), according to which the NK in 3D is given by

$$
V_{\text {kick }}=60 \frac{\mathrm{km}}{\mathrm{s}}\left(\frac{M_{\text {ejecta }}}{M_{\mathrm{NS} / \mathrm{BH}}}\right)+130 \mathrm{~km} \mathrm{~s}^{-1} \text {, }
$$

where $M_{\text {ejecta }}$ is the mass ejected from the star during the SN and $M_{\mathrm{NS} / \mathrm{BH}}$ is the mass of a resulting NS or a BH. Although Bray \& Eldridge (2018) provided their fit only for NSs, we apply it also to BHs as a parameter study.

\subsection{Mergers of Binary Components}

Stellar mergers may constitute a significant fraction of massive stars (e.g., Langer 2012) and, therefore, progenitors of BHs. Head-on collisions may play a significant role in dense stellar systems like globular clusters (e.g., Glebbeek et al. 2013), but stellar mergers in field populations are more probable, due to loss of orbital angular momentum (e.g., during the CE phase). Podsiadlowski et al. (1992) predicted that $\sim 10 \%$ of $8<M<20 M_{\odot}$ primaries merge with their companions before an SN, and de Mink et al. (2014) showed that $\sim 8_{-4}^{+9} \%$ of massive single stars may actually be products of binary mergers. Objects like Red Novae or Blue Stragglers are thought to be observed during or after the merging process (e.g., Leonard 1989; Kochanek et al. 2014; Blagorodnova et al. 2017).

In the case of non-DCO mergers (including mergers of a compact object with a noncompact object), a merger process is not well understood. Especially, the amount of mass ejected from the system, which is an important factor for postmerger evolution, is poorly constrained. A product of a merger of two MS stars may evolve similarly to a star that was always single, but if evolved stars are involved, the postmerger evolution is more complicated (Glebbeek et al. 2013). Especially, a merger outcome may evolve unlike any single star (Vigna-Gómez et al. 2019).

We assume that non-DCO mergers occur in the following situations:

1. There is a failed envelope ejection during a $\mathrm{CE}$ event (e.g., Justham et al. 2014).

2. The donor in a CE phase has not developed a clear boundary between core and envelope (e.g., MS and HG donors; Ivanova \& Taam 2004).

3. The donor's radius exceeds two times its Roche lobe radius $\left(R_{\text {donor }}>2 R_{\text {donor, } \mathrm{RL}}\right)$ during $\mathrm{RLOF}$.

Because the merger physics and postmerger evolution are poorly understood, we provide information on the binary parameters just before the merger to allow for different approaches to this conundrum.

As far as DCO mergers are concerned, many studies were devoted to an in-depth analysis of this phenomenon in stellar populations (e.g., Lipunov et al. 1997a; Dominik et al. 2012, 2013, 2015; Mennekens \& Vanbeveren 2014; Belczynski et al. 2016b; Mandel \& de Mink 2016). DCO mergers affect the BH populations, especially the $\mathrm{BH}$ mass distribution. As a part of our results, in the public database we provide information about DCO formation and estimate the time to merger using the formula of Peters (1964). In order to give predictions and estimate the fraction of BHs that come from DCO mergers, we assumed that the mass loss during a DCO merger is negligible.

In this work, in order to estimate the population of SBHs originating from binary mergers, we adopt the simplistic approach of Olejak et al. (2019). We include only the main channels responsible for $>95 \%$ of all mergers. Specifically, for different evolutionary types of stars, we use the following procedure:

1. MS+MS: Outcome is an MS star. We assume that half of the mass of the lighter component is lost in the process.

2. MS+HeS: Outcome is a helium star $(\mathrm{HeS})$. We assume that half of the mass of the MS component is lost in the process. 
3. HeS+HeS: Outcome is a HeS. We assume that half of the mass of the lighter component is lost in the process.

4. NS+MS/HeS: We assume that half of the mass of the MS/HeS star is lost in the process and that it becomes an NS/BH if its final mass is lower/higher than $M_{\max , \mathrm{NS}}=2.5 M_{\odot}$.

5. $\mathrm{BH}+\mathrm{MS} / \mathrm{HeS}$ : We assume that half of the mass of the $\mathrm{MS} / \mathrm{HeS}$ star is lost in the process and that it becomes a BH.

6. $\mathrm{BH}+\mathrm{NS} / \mathrm{BH}$ : The outcome is a $\mathrm{BH}$ with a mass equal to the total mass of the binary before the merger.

We neglect other types of mergers because we found that they constitute only a small fraction $(\lesssim 5 \%)$ of all mergers, and predictions for their outcomes are even less certain. For postmerger MS and HeSs, we calculate their further evolution assuming that they are on the ZAMS or the zero-age helium main sequence, respectively, in order to find $\mathrm{BH}$ predecessors and calculate a compact object's mass. We note that the presented approach is only quantitative, but it helps us to estimate the importance of stellar mergers for $\mathrm{BH}$ populations.

\subsection{General Simulation Properties}

In this work, we concentrate on a description of $\mathrm{BH}$ populations originating from massive binaries, which we define as binaries with a primary's (i.e., the heavier star on ZAMS) initial mass $M_{\text {ZAMS, a }}>10 M_{\odot}$. Our analysis does not include BHs in triples and higher-order systems (e.g., Antonini et al. 2017) or BHs in binaries formed from stellar encounters in dense stellar systems (e.g., Banerjee 2017).

The total simulated stellar mass is $4.8 \times 10^{8} M_{\odot}$ for the STD model and $1.1 \times 10^{9} M_{\odot}\left(2.4 \times 10^{8} M_{\odot}\right)$ for the steep IMF (flat IMF) model. Throughout this paper, we assume a $50 \%$ binary fraction on ZAMS for low-mass stars $\left(M_{\text {ZAMS }}<10 M_{\odot}\right)$ and a $100 \%$ binary fraction for heavier stars $\left(M_{\text {ZAMS }} \geqslant 10 M_{\odot}\right)$. The simulated stellar mass corresponds to about $\sim 0.8 \%$ (1.9\% for steep IMF model or $0.4 \%$ for flat IMF model) of the stellar mass of the MW equivalent galaxy (MWEG; $M_{\mathrm{MW}} \approx 6 \times 10^{10} M_{\odot}$; e.g., Licquia \& Newman 2015).

For the purpose of our presentation, all results are scaled to the stellar mass of an MWEG $\left(M_{\mathrm{MW}} \approx 6 \times 10^{10} M_{\odot}\right.$; Licquia \& Newman 2015), and the SFH is chosen to be constant through the last $10 \mathrm{Gyr}\left(\mathrm{SFR}=6 M_{\odot} \mathrm{yr}^{-1}\right)$. Such a model, although simple, allows us to draw general conclusions. Raw results may be scaled by using any realistic total stellar mass, and any SFH may be applied. In general, the changes in SFH are not as important as the total mass of formed stars, as there is only a small delay time between the formation of a binary (ZAMS) and the formation of a $\mathrm{BH}$ (typically less than a few $\times 10 \mathrm{Myr}$ ), which are, typically, single. We note that for a part of the BH population, the SFH may, actually, be important, such as for DCOs, which have steep time-to-merger distributions $\left(t_{\text {merge }} \propto t^{-1}\right.$; e.g., Dominik et al. 2012). Additionally, if the spatial distribution of $\mathrm{BHs}$ is concerned, the change in SFH may play a role, because it affects the time that $\mathrm{BHs}$ have to spread throughout the space. What is more, BHs formed earlier have more time to be ejected from the stellar systems, due to their NKs or as a result of dynamical interactions. Deeper analyses of changes in spatial distributions, SFH, and chemical evolution are left for separate studies (e.g., Olejak et al. 2019).

\section{Results}

The main results of the simulations are summarized in Table 2 and the mass distribution of all BHs in the STD model is present in Figure 1. Most of the BHs are predicted to exist as SBHs either as a result of a binary disruption $(\mathrm{dSBH})$ or a stellar merger (mSBH; with a BH involved, or producing a star massive enough to form a $\mathrm{BH}$ ). The population of $\mathrm{BHs}$ in binaries (both in DCOs and in BHBs) is about an order of magnitude smaller and in the case of models with increased $\mathrm{BH}$ NKs $\left(\mathrm{NK}_{\mathrm{R}}\right.$ and $\mathrm{NK}_{\mathrm{BE}}$ ) even two orders of magnitude smaller. Only a very small fraction of the BHBs are the interacting ones (see Section 3.2.3).

The initial separation influences significantly the fate of a massive binary. In general, if the separation is very large $\left(a \gtrsim 3000 R_{\odot}\right)$, the binary will mostly disrupt and produce SBHs (Section 3.1). A low separation $\left(a_{\text {ZAMS }} \lesssim 30 R_{\odot}\right)$ will lead frequently to a non-DCO merger (Section 3.3), which may be massive enough to form an SBH. Objects with medium initial separations have a much more uncertain fate and may merge, become disrupted, or form a bound binary with at least one BH inside (Section 3.2). In the case of a binary harboring a $\mathrm{BH}$, the system after some time may still merge (e.g., as a DCO merger, Section 3.2.1) or be disrupted during the formation of a second compact object (e.g., $\mathcal{R}_{\mathrm{dSBH}, 2}$ formation route, Section 3.1.1).

\subsection{Single BHs from Disrupted Binaries}

If a system hosting a $\mathrm{BH}$ progenitor becomes disrupted, which may occur before, after, or during the $\mathrm{BH}$ formation, a compact object becomes a dSBH (we use "dSBH" to represent an SBH from a disrupted binary, as SBHs can also form from mergers; see Section 3.3). Binaries may become disrupted by an NK that one component obtains after an SN explosion, due to a Blaauw kick (e.g., because of a significant loss of mass, $\gtrsim 50 \% M_{\text {tot }}$, from a binary in an SN explosion; Blaauw 1961) or interaction with a third star. Although in our simulations we included only isolated binaries, we note that interactions, even in sparse stellar systems like the Galactic disk, may significantly alter the binary evolution (Kaib \& Raymond 2014; Klencki et al. 2017).

We found out that the tested initial parameter distributions have little effect on the properties of the resulting population of dSBHs. The steepness of the IMF changes the number of resulting dSBHs by $\Delta N_{\mathrm{dSBH}} \approx 70 \% / 160 \%$, due to the decreased/increased relative number of $\mathrm{BH}$ progenitors for steep IMF/flat IMF models. The effect of metallicity and NK models is not as strong $\left(\Delta N_{\mathrm{dSBH}} \approx 40 \%\right.$ and $20 \%$, respectively).

\subsubsection{Typical Evolutionary Routes}

We found that two evolutionary routes (see Table 3) dominate most of the dSBH production and are common for all models. The number of dSBHs produced through these routes and for different models is presented in Table 4. Both channels involve a binary initially composed of two massive stars that will undergo SN explosions or collapse directly into BHs. The former case may lead to a binary disruption (due to NK or mass loss). The main difference between the routes comes from the initial separation. In the case of $\mathcal{R}_{\mathrm{dSBH}, 1}$, the initial distance between stars is lower than $\sim 1000 R_{\odot}$, whereas for $\mathcal{R}_{\mathrm{dSBH}, 2}$, it is usually larger then $\sim 5000 R_{\odot}$. Consequently, 
Table 2

Number of BHs in the Milky Way Equivalent Galaxy

\begin{tabular}{|c|c|c|c|c|c|c|c|c|c|}
\hline \multirow{2}{*}{$\frac{\text { Model }}{\text { STD }}$} & \multirow{2}{*}{$\frac{N_{\mathrm{BH}, \text { tot }}}{1.1 \times 10^{8}}$} & \multicolumn{2}{|c|}{$N_{\mathrm{dSBH}}$} & \multicolumn{2}{|c|}{$N_{\text {BHB }}$} & \multicolumn{2}{|c|}{$N_{\text {BH,DCO }}$} & \multicolumn{2}{|c|}{$N_{\mathrm{mSBH}}$} \\
\hline & & $5.4 \times 10^{7}$ & $(49.7 \%)$ & $1.5 \times 10^{6}$ & $(1.4 \%)$ & $5.1 \times 10^{6}$ & $(4.7 \%)$ & $4.8 \times 10^{7}$ & $(44.2 \%)$ \\
\hline mid- $Z$ & $1.1 \times 10^{8}$ & $3.4 \times 10^{7}$ & $(30.6 \%)$ & $2.0 \times 10^{6}$ & $(1.8 \%)$ & $1.2 \times 10^{7}$ & $(10.8 \%)$ & $6.3 \times 10^{7}$ & $(56.8 \%)$ \\
\hline low-Z & $1.2 \times 10^{8}$ & $3.7 \times 10^{7}$ & $(30.0 \%)$ & $1.5 \times 10^{6}$ & $(1.2 \%)$ & $1.9 \times 10^{7}$ & $(15.4 \%)$ & $6.6 \times 10^{7}$ & $(53.4 \%)$ \\
\hline SS0 & $1.1 \times 10^{8}$ & $6.6 \times 10^{7}$ & $(58.8 \%)$ & $3.4 \times 10^{6}$ & $(3.0 \%)$ & $6.9 \times 10^{6}$ & $(6.1 \%)$ & $3.6 \times 10^{7}$ & $(32.1 \%)$ \\
\hline $\mathrm{NK}_{\mathrm{R}}$ & $1.1 \times 10^{8}$ & $6.4 \times 10^{7}$ & $(58.1 \%)$ & $5.9 \times 10^{4}$ & $(0.1 \%)$ & $1.1 \times 10^{4}$ & $(0.0 \%)$ & $4.6 \times 10^{7}$ & $(41.8 \%)$ \\
\hline $\mathrm{NK}_{\mathrm{BE}}$ & $1.1 \times 10^{8}$ & $6.5 \times 10^{7}$ & $(58.5 \%)$ & $5.4 \times 10^{4}$ & $(0.0 \%)$ & $2.7 \times 10^{3}$ & $(0.0 \%)$ & $4.6 \times 10^{7}$ & $(41.4 \%)$ \\
\hline flat IMF & $2.7 \times 10^{8}$ & $1.4 \times 10^{8}$ & $(52.6 \%)$ & $3.2 \times 10^{6}$ & $(1.2 \%)$ & $1.3 \times 10^{7}$ & $(4.9 \%)$ & $1.1 \times 10^{8}$ & $(41.3 \%)$ \\
\hline steep IMF & $3.4 \times 10^{7}$ & $1.6 \times 10^{7}$ & $(46.8 \%)$ & $5.6 \times 10^{5}$ & $(1.6 \%)$ & $1.6 \times 10^{6}$ & $(4.7 \%)$ & $1.6 \times 10^{7}$ & $(46.8 \%)$ \\
\hline
\end{tabular}

Note. Number of BHs for different tested models (see Table 1). Values are presented for a simple MW model with a total simulated stellar mass of $6 \times 10^{10} M_{\odot}$ and constant star formation during the last $10 \mathrm{Gyr}$. Column headers stand for $N_{\mathrm{BH}, \mathrm{tot}}$, total estimated number of BHs; $N_{\mathrm{dSBH}}$, number of single BHs from disrupted binaries; $N_{\mathrm{BHB}}$, number of binaries harboring a BH and a noncompact companion; $N_{\mathrm{BH}, \mathrm{DCO}}$, number of BHs in DCOs (BH+BH is counted as two BHs; see Table 5); $M_{\mathrm{mSBH}}$, a rough estimate of the number of stellar mergers (including DCO) that will be massive enough to form a BH (see Section 2.2).

Table 3

Typical Formation Routes of BHs

\begin{tabular}{lc}
\hline \hline Route & \multicolumn{1}{c}{ Evolutionary Route } \\
\hline $\mathcal{R}_{\mathrm{dSBH}, 1}$ & Single BHs from disrupted binaries \\
$\mathcal{R}_{\mathrm{dSBH}, 2}$ & $\mathrm{MT} 1(1 / 2 / 4 / 5-1 / 2 / 4 / 5) \mathrm{SN} 1$ Disruption SN2 \\
& $\mathrm{S} 1 \mathrm{SN} 2$ Disruption \\
& $\mathrm{BH}$ XRBs \\
$\mathcal{R}_{\mathrm{MTBHB}, 1}$ & $\mathrm{CE} 1(4 / 5-1 ; 7 / 8-1) \mathrm{SN} 1 \mathrm{MT} 2(14-1 / 2 / 3 / 4 / 5 / 6)$ \\
$\mathcal{R}_{\mathrm{MTBHB}, 2}$ & $\mathrm{CE} 1(4 / 5-1 ; 7 / 8-1) \mathrm{SN} 1 \mathrm{MT} 2(13-1 / 2 / 3) \mathrm{AICBH} 1$ \\
& $\mathrm{MT} 2(14-1 / 2 / 3)$ \\
& $\mathrm{DCOs}$ \\
$\mathcal{R}_{\mathrm{DCO}, 1}$ & $\mathrm{SN} 1 \mathrm{SN} 2$ \\
$\mathcal{R}_{\mathrm{DCO}, 2}$ & $\mathrm{MT} 1(1 / 2 / 4 / 5-1 / 2 / 4) \mathrm{SN} 1$ \\
& $\mathrm{CE} 2(13 / 14-4 / 5 ; 13 / 14-7 / 8) \mathrm{SN} 2$ \\
& $\mathrm{CE} 1(2 / 3 / 4-1 ; 7-1) \mathrm{MT} 1(7-1)$ Merger \\
$\mathcal{R}_{\mathrm{mSBH}}$ &
\end{tabular}

Note. Schematic representations of the evolutionary routes for typical BH formation channels. Wide BHBs (Section 3.2.2) are not included in the table as their evolution does not include any interactions and their evolutionary route is "SN1" by definition. Details of the postmerger evolution are not included in our study. Symbols in evolutionary routes are as follows: SN1/2, supernova of the primary/secondary; MT1 $/ 2$, mass transfer (primary/secondary is a donor); CE1/2, common envelope (primary/secondary is a donor; numbers in parentheses represent typical primary evolutionary type (left) and secondary (right); the first two numbers represent initial types (prior to CE), whereas the last two numbers represent the final types (after CE)); AICBH1, accretioninduced collapse of an NS primary to a BH (assumed to occur after an NS obtains the mass $M \geqslant M_{\text {max,NS }}=2.5 M_{\odot}$ ). Evolutionary types (numbers inside parentheses) are as follows: 1 , main sequence; 2 , Hertzsprung gap; 3 , red giant; 4, core helium burning; 5, early asymptotic giant branch; 6 , thermal pulsing asymptotic giant branch; 7, helium main sequence; 8, evolved helium star; 13, neutron star; 14, black hole.

the former route leads through an interaction phase (typically the MT), whereas a binary evolving through the latter one experiences no interactions.

For a typical binary evolving through the $\mathcal{R}_{\mathrm{dSBH}, 1}$ route (Figure 2, upper plot), the primary is about $26 M_{\odot}$ on the ZAMS, whereas the secondary is slightly lighter $\left(\sim 21 M_{\odot}\right)$. The separation is modest $\left(\sim 300 R_{\odot}\right)$. The primary evolves faster and after $\sim 6.7 \mathrm{Myr}$, while expanding as an HG star, fills
Table 4

Single BHs from Disrupted Binaries

\begin{tabular}{|c|c|c|c|}
\hline Model & Route & Number & $a_{\mathrm{ZAMS}}\left[R_{\odot}\right]$ \\
\hline \multirow[t]{2}{*}{ STD } & $\mathcal{R}_{\mathrm{dSBH}, 1}$ & $3.6 \times 10^{7}(67 \%)$ & $28-400$ \\
\hline & $\mathcal{R}_{\mathrm{dSBH}, 2}$ & $1.7 \times 10^{7}(32 \%)$ & $>4400$ \\
\hline \multirow[t]{2}{*}{ mid- $Z$} & $\mathcal{R}_{\mathrm{dSBH}, 1}$ & $2.0 \times 10^{7}(58 \%)$ & $20-800$ \\
\hline & $\mathcal{R}_{\mathrm{dSBH}, 2}$ & $1.2 \times 10^{7}(33 \%)$ & $>7700$ \\
\hline \multirow[t]{2}{*}{ low-Z } & $\mathcal{R}_{\mathrm{dSBH}, 1}$ & $1.5 \times 10^{7}(40 \%)$ & $17-180$ \\
\hline & $\mathcal{R}_{\mathrm{dSBH}, 2}$ & $2.0 \times 10^{7}(55 \%)$ & $>1600$ \\
\hline \multirow[t]{2}{*}{ SS0 } & $\mathcal{R}_{\mathrm{dSBH}, 1}$ & $4.0 \times 10^{7}(61 \%)$ & $28-1000$ \\
\hline & $\mathcal{R}_{\mathrm{dSBH}, 2}$ & $2.4 \times 10^{7}(36 \%)$ & $>6200$ \\
\hline \multirow[t]{2}{*}{$\mathrm{NK}_{\mathrm{R}}$} & $\mathcal{R}_{\mathrm{dSBH}, 1}$ & $4.0 \times 10^{7}(62 \%)$ & $28-400$ \\
\hline & $\mathcal{R}_{\mathrm{dSBH}, 2}$ & $2.2 \times 10^{7}(35 \%)$ & $>5200$ \\
\hline \multirow[t]{2}{*}{$\mathrm{NK}_{\mathrm{BE}}$} & $\mathcal{R}_{\mathrm{dSBH}, 1}$ & $4.1 \times 10^{7}(63 \%)$ & $28-400$ \\
\hline & $\mathcal{R}_{\mathrm{dSBH}, 2}$ & $2.2 \times 10^{7}(34 \%)$ & $>5200$ \\
\hline \multirow[t]{2}{*}{ flat IMF } & $\mathcal{R}_{\mathrm{dSBH}, 1}$ & $9.4 \times 10^{7}(66 \%)$ & $28-430$ \\
\hline & $\mathcal{R}_{\mathrm{dSBH}, 2}$ & $4.7 \times 10^{7}(33 \%)$ & $>5400$ \\
\hline \multirow[t]{2}{*}{ steep IMF } & $\mathcal{R}_{\mathrm{dSBH}, 1}$ & $1.1 \times 10^{7}(68 \%)$ & $28-400$ \\
\hline & $\mathcal{R}_{\mathrm{dSBH}, 2}$ & $5.1 \times 10^{6}(31 \%)$ & $>5000$ \\
\hline
\end{tabular}

Note. Number of single BHs originating from disrupted binaries. Only the main evolutionary routes are included. The initial separation $\left(a_{\text {ZAMS }}\right)$ is the main differing factor between the main routes. See Section 3.1.1 for details.

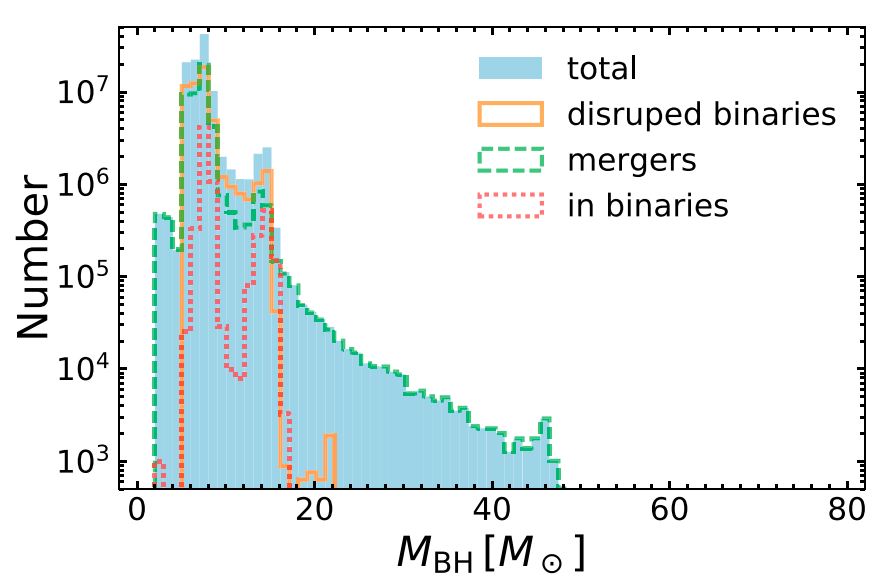

Figure 1. BH mass distribution for the STD model containing single BHs from disrupted binaries and mergers and $\mathrm{BHs}$ residing in binaries. The results are scaled for the MWEG with a total stellar mass of $6 \times 10^{10} M_{\odot}$ and constant SFR throughout the last $10 \mathrm{Gyr}$. 


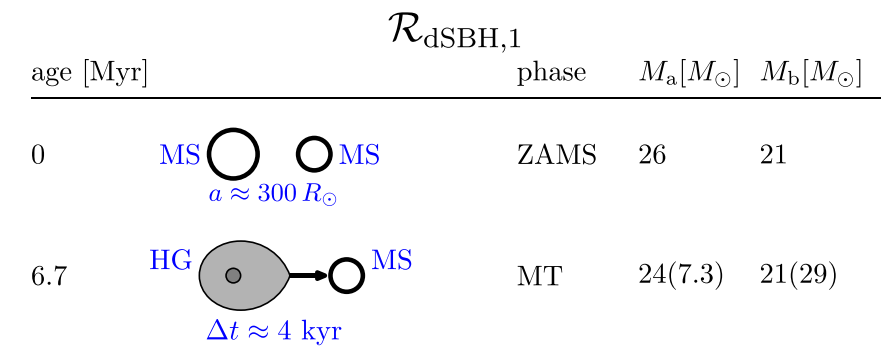

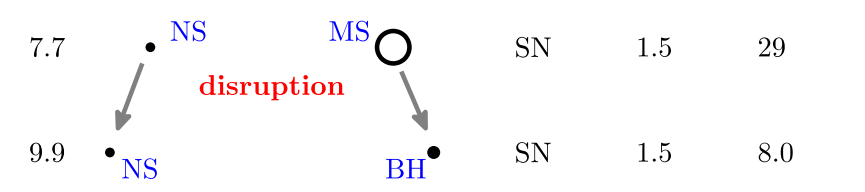

\begin{tabular}{lllll}
\multicolumn{5}{c}{$\mathcal{R}_{\mathrm{dSBH}, 2}$} \\
age $[\mathrm{Myr}]$ & phase & $M_{\mathrm{a}}\left[M_{\odot}\right]$ & $M_{\mathrm{b}}\left[M_{\odot}\right]$ \\
\hline 0 & $\mathrm{MS} \bigcup_{a \approx 5,000 R_{\odot}}$ & ZAMS & 25 & 10
\end{tabular}

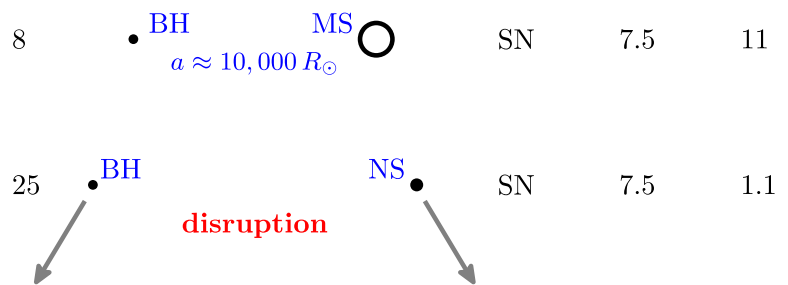

Figure 2. Schematic representations of the typical evolutionary scenarios leading to the formation of single BHs originating from disrupted binaries (dSBH; Section 3.1.1). "Age" represents the time since ZAMS, and $M_{\mathrm{a}} / M_{\mathrm{b}}$ stands for the mass of the primary/secondary. The evolutionary phases appearing in the figure are the following: ZAMS, zero-age main sequence; MT, mass transfer; SN, supernova, that is, compact object formation. Highlighted stages in stellar evolution are as follows: MS, main sequence; HG, Hertzsprung gap; NS, neutron star; $\mathrm{BH}$, black hole. The numbers in parentheses represent the masses at the end of the MT phase.

its Roche lobe, and MT begins onto the secondary, which is still on its MS. Although the MT is relatively short ( $\sim 4 \mathrm{kyr})$, the primary loses its hydrogen envelope and becomes a $\sim 7 M_{\odot}$ helium star. Half of the expelled envelope is accreted by the secondary, which grows to $\sim 29 M_{\odot}$ while still being on its MS. After about $1 \mathrm{Myr}$, the primary goes through $\mathrm{SNIb} / \mathrm{c}$ and becomes a $\sim 1.5 M_{\odot}$ NS. An NK disrupts the system. Due to its significant mass, the velocity obtained by the secondary is very low $\left(\sim 5 \mathrm{~km} \mathrm{~s}^{-1}\right)$. The massive secondary needs only $\sim 2 \mathrm{Myr}$ to become a BH with a mass of $\sim 8 M_{\odot}$. The NK in a case of such a $\mathrm{BH}$ is negligible, so its velocity does not change significantly. It is noteworthy that most of the BHs formed through the $\mathcal{R}_{\mathrm{dSBH}, 1}$ route originate from secondary stars, that is, less massive ones on the ZAMS.

The $\mathcal{R}_{\mathrm{dSBH}, 1}$ channel is similar to the one proposed for the formation of single massive stars (potential $\mathrm{BH}$ progenitors) by Renzo et al. (2019). Using a different population synthesis code, they found that $86_{-9}^{+11} \%$ (depending on the model parameters) of binaries evolving through this channel will become disrupted during the first $\mathrm{SN}$.

In the other typical route $\left(\mathcal{R}_{\mathrm{dSBH}, 2}\right.$; Figure 2 , bottom plot), the primary is about $25 M_{\odot}$ on ZAMS, whereas the secondary

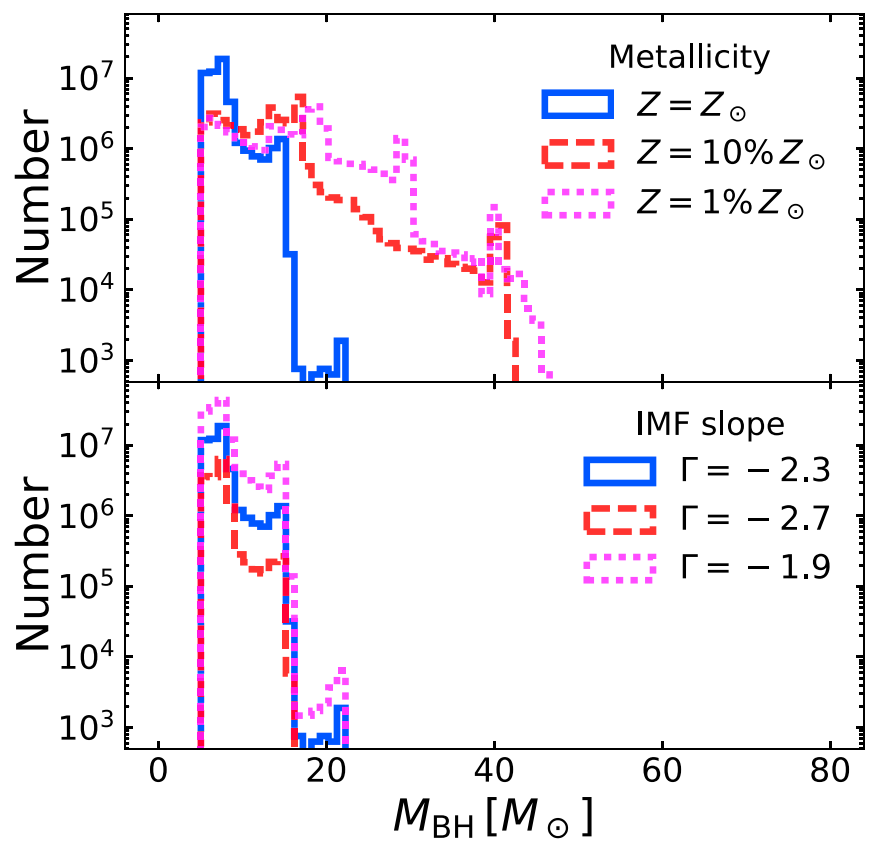

Figure 3. Mass distribution of the single BHs from disrupted binaries. The locations of the main peaks depend on metallicity (upper plot; e.g., $\sim 7-8 M_{\odot}$ and $\sim 15 M_{\odot}$ for STD model, and $\sim 15-30 M_{\odot}$ and $\sim 40 M_{\odot}$ for mid- $Z$ and low- $Z$ models). We note that the peak at $\sim 40 M_{\odot}$ in the mid- $Z$ model results from mass loss in stellar winds, whereas in the low- $Z$ model it is a consequence of pair-pulsation SNe. Two main evolutionary routes, $\mathcal{R}_{\mathrm{dSBH}, 1}$ and $\mathcal{R}_{\mathrm{dSBH}, 2}$, have similar mass distributions with the peaks located at the same masses for all models with the same metallicity. All distributions reveal a highmass extension (a peak at $\sim 22 M_{\odot}$ for the STD model and a tail at $\sim 40-45 M_{\odot}$ for models mid- $Z$ and low-Z), which is a result of the mass accretion onto a $\mathrm{BH}$, or its progenitor, prior to disruption.

is $\sim 10 M_{\odot}$. The initial separation is as large as $\sim 6000 R_{\odot}$. As a consequence, their Roche lobes are huge, and no interaction is possible throughout their evolution. The heavier star evolves faster and in $\sim 8 \mathrm{Myr}$ forms a $\sim 7.5 M_{\odot}$ BH with a small NK. The separation grows to $\sim 10,000 R_{\odot}$, which results from a loss of mass in the stellar wind from both stars. After an additional $\sim 17 \mathrm{Myr}$, the secondary explodes as an SN and forms an NS. A strong NK disrupts the system. Due to a large mass ratio between compact objects $(q \approx 7)$, the postdisruption velocity of the BH is relatively small $\left(\sim 5 \mathrm{~km} \mathrm{~s}^{-1}\right)$.

\subsubsection{Mass Distribution of $d S B H s$}

The dSBH mass distribution is presented in Figure 3. The shape is similar to the distribution of $\mathrm{BH}$ masses in singlestar evolution (compare, e.g., $M_{\text {remnant }}\left(M_{\text {ZAMS }}\right)$ relations in Belczynski et al. 2010a). The main peak $\left(\sim 7-8 M_{\odot}\right.$ for solar metallicity (STD model; $Z=Z_{\odot}$ ) or $\sim 15-30 M_{\odot}$ for lower metallicities (models mid- $Z$ and low- $Z ; Z=10 \% Z_{\odot}$ and $Z=1 \% Z_{\odot}$, respectively)) relates to $\mathrm{BHs}$ formed from $\sim 20-35 M_{\odot}$ progenitors through failed SN explosion (e.g., Belczynski et al. 2012; Fryer et al. 2012). The second peak ( $\sim 15 M_{\odot}$ for STD or $\sim 40 M_{\odot}$ for mid-Z model) comes from BHs originating from the most massive stars $\left(\gtrsim 100 M_{\odot}\right)$ on ZAMS, which lose a large part of their mass in the stellar wind. In low-metallicity environments (model low- $Z$ ), the peak results from the pair-pulsation SN (e.g., Belczynski et al. 2016a), which prevents the formation of heavier BHs in singlestar evolution. The third peak $\left(\sim 22 M_{\odot}\right.$ for STD, or the tail 
extending to $\sim 45 M_{\odot}$ for mid- $Z$ and low- $Z$ ) is formed through binary interactions and is not present in distributions for singlestar evolution (compare Belczynski et al. 2010a, 2016a).

The similarity of the mass distributions of $\mathrm{BHs}$ for single stars and binaries is a direct consequence of the fact that massive binaries, which later on became disrupted, usually interact only little (the mass of the $\mathrm{BH}$ progenitor is not changed significantly; $\left.\mathcal{R}_{\mathrm{dSBH}, 1}\right)$ or not at all $\left(\mathcal{R}_{\mathrm{dSBH}, 2}\right)$, so in most cases the binary components evolve as in isolation.

A notable difference is the presence of the high-mass peak and tail in the mass distributions. It is most prominent for the models with solar metallicity where the tail extends up to $\sim 22 M_{\odot}$, whereas the heaviest BHs forming at that metallicity from single stars are only $\sim 15 M_{\odot}$ (Belczynski et al. 2010a). These massive SBHs constitute only a tiny fraction of the population $(<1 \%)$. Typically, the initial total mass of a system in which such overmassive BHs are formed is above $200 M_{\odot}$, and the mass ratio is $\sim 1$. The primary, being slightly heavier, is the first to expand and fill its Roche lobe while being on an HG. At the moment of interaction, both stars have masses of about $60 M_{\odot}$ due to strong mass losses in the stellar wind. When the mass transfer ceases, the primary becomes a $\sim 25 M_{\odot}$ core helium burning $(\mathrm{CHeB})$ star. The secondary, being now much heavier $\left(\sim 80 M_{\odot}\right)$, appears rejuvenated (e.g., Tout et al. 1997), evolves much faster, and after $\sim 400 \mathrm{kyr}$ forms a BH first with a mass of $\sim 21 M_{\odot}$. The primary needs an additional $100 \mathrm{kyr}$ to become a 7.3 $M_{\odot} \mathrm{BH}$. During the second $\mathrm{SN}$, the NK easily disrupts a wide $\left(\sim 15,000 R_{\odot}\right)$ binary.

\subsubsection{Significance of Metallicity}

The relation between the number of dSBHs and metallicity was found to be nonmonotonic. The largest number of dSBHs with respect to metallicity is produced in the STD model $\left(5.4 \times 10^{7}\right)$, whereas the smallest is in the mid- $Z$ model $\left(3.4 \times 10^{7}\right)$. For the low- $Z$ model, the number of dSBHs is slightly higher $\left(3.7 \times 10^{7}\right)$ than in the mid- $Z$ model.

The number of dSBHs produced through the $\mathcal{R}_{\mathrm{dSBH}, 1}$ route increases monotonically with metallicity. The higher the metallicity, the stronger is the line-driven stellar wind. Only a fraction of this mass may be accreted by a companion, so the bulk of it leaves the system, which results in orbital expansion. Consequently, the stronger the stellar wind, the wider the binary becomes. Simultaneously, the stronger mass loss also makes the companion lighter during the NS formation. Wider orbits and lighter companions make the system easier to disrupt, which produces more SBHs.

A different mechanism results in the nonmonotonic relation between the metallicity and the number of BHs produced through the $\mathcal{R}_{\mathrm{dSBH}, 2}$ route. Metallicity affects the evolutionary expansion of the stars, which happens to be the strongest in the mid- $Z$ environment and the smallest in the low- $Z$ environment (Figure 4). Only binaries with separations high enough to avoid Roche lobe filling may evolve without interactions. The number of such systems will be smaller if the nuclear expansion is on average higher, as in the case of the mid- $Z$ model.

\subsubsection{Initial BH Velocities and High-velocity dSBHs}

When an SBH forms (after disruption of the binary or BH formation, whichever happens later), its velocity is not only a

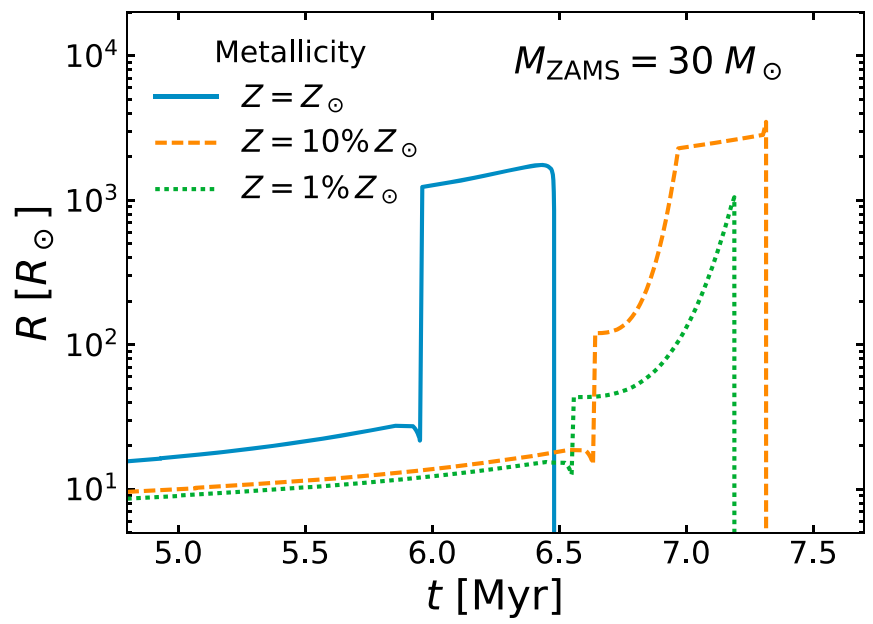

Figure 4. Radius evolution of a star with a ZAMS mass of $M_{\text {ZAMS }}=$ $30 M_{\odot}$ for three different metallicities. The largest radii are obtained for moderate metallicity $\left(Z=10 \% Z_{\odot}\right.$; mid- $Z$ model $)$, whereas they are the smallest for low metallicity $\left(Z=1 \% Z_{\odot} ;\right.$ low- $Z$ model $)$.

result of the motion in the gravitational potential (e.g., of a galaxy) but also preceding evolutionary processes, like NK, Blaauw kick, and binary disruption. The velocity that is the result of the latter processes calculated at the moment of the binary disruption or the formation of the $\mathrm{BH}$ (whichever happens later) we call here an initial BH's velocity $\left(v_{\mathrm{BH}, 0}\right)$. Such a definition, in which we do not involve the motion in the gravitational potential, allows for application of our results to any gravitational potential (e.g., different galaxies or different models of the MW galaxy). In this work, we provide the magnitudes of 3D velocities (i.e., lengths of the velocity vectors) and assume that the distribution of NKs is isotropic. We note that, in a realistic situation, the velocity of a dSBH will be modified by the gravitational potential of a stellar system and interactions with other stars. Although these effects may also affect the binary evolution, the short $\mathrm{dSBH}$ formation timescale $(\lesssim 50 \mathrm{Myr})$ allows us to assume that the effect is usually negligible.

The highest values of $v_{\mathrm{BH}, 0}$ are obtained for $\mathrm{BHs}$ with the highest NKs. We found that the majority of dSBHs with the highest initial velocities $\left(v_{\mathrm{BH}, 0}>300 \mathrm{~km} \mathrm{~s}^{-1}\right)$ within the highest $10 \%$ were formed through the $\mathcal{R}_{\mathrm{dSBH}, 1}$ route and involve the lowest $\mathrm{BH}$ masses and the shortest predisruption periods. We note that Renzo et al. (2019) found that the majority of the secondaries $(\sim 90 \%)$ after the disruption will have low velocities $\left(\lesssim 20 \mathrm{~km} \mathrm{~s}^{-1}\right)$, which agrees with our predictions for the standard NK model.

The $v_{\mathrm{BH}, 0}$ distributions for all tested NK models are presented in Figure 5. The maximal $v_{\mathrm{BH}, 0}$ of a dSBH may reach $\sim 500 \mathrm{~km} \mathrm{~s}^{-1}$ for the STD model and nearly $\sim 1000 \mathrm{~km} \mathrm{~s}^{-1}$ for the low- $Z$ model. The higher velocities for lower metallicities are the result of the formation of closer binaries through the $\mathcal{R}_{\mathrm{dSBH}, 1}$ route, which, together with higher masses of $\mathrm{BHs}$, gives larger orbital speeds (see also Renzo et al. 2019).

A distribution of $v_{\mathrm{BH}, 0}$ for the standard NK prescription (e.g., STD model; Hobbs et al. 2005) has two distinctive parts (Figure 5). The low-velocity peak is connected with BHs formed with no NK due to heavy fallback, which, as we assume, decreases the $\mathrm{NK}$, or in a direct collapse associated with no NK. The wide high-velocity component comprises 


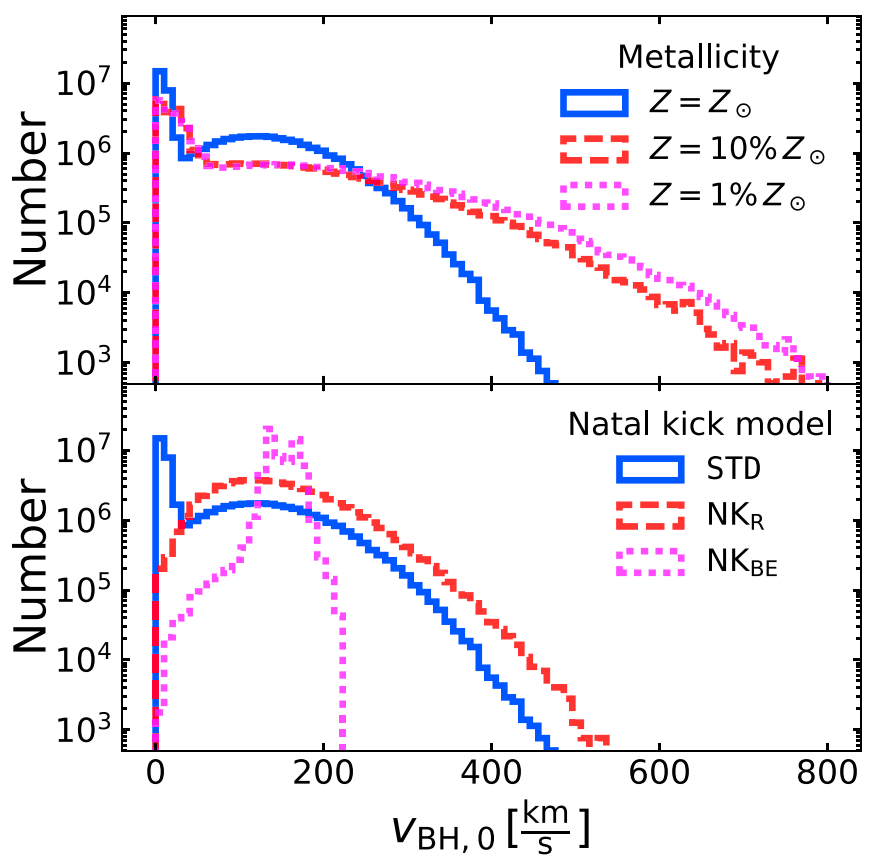

Figure 5. Distributions of initial velocities $\left(v_{\mathrm{BH}, 0}\right)$ of single $\mathrm{BHs}$ originating from disrupted binaries. All models with a standard (Hobbs et al. 2005) NK prescription have a similar bimodal distribution. $\mathrm{NK}_{\mathrm{R}}$ and $\mathrm{NK}_{\mathrm{BE}} \mathrm{NK}$ prescriptions give significantly different distributions. NKs become larger for lower metallicities, resulting in wider initial velocity distributions. We note that the local escape velocity from the Milky Way (at radius $\sim 8.3 \mathrm{kpc}$ ) is $\sim 520 \mathrm{~km}$ $\mathrm{s}^{-1}$ and drops to $\sim 380 \mathrm{~km} \mathrm{~s}^{-1}$ at radius $50 \mathrm{kpc}$ (e.g., Williams et al. 2017).

BHs that are much lighter, thus formed with no significant fallback. Models $\mathrm{NK}_{\mathrm{R}}$ and $\mathrm{NK}_{\mathrm{BE}}$ lead to a number of dSBHs $\left(6.4 \times 10^{7}\right.$ and $\left.6.5 \times 10^{7}\right)$ comparable to that of the STD model $\left(5.4 \times 10^{7}\right)$, but the shape of the $v_{\mathrm{BH}, 0}$ distribution is significantly different. The $\mathrm{NK}_{\mathrm{R}}$ model lacks a low-velocity peak as the NK is independent of the fallback. Therefore, the average NK is much higher than in the STD model and typically equals $\sim 125 \mathrm{~km} \mathrm{~s}^{-1}$ for a typical BH mass of $7.5 M_{\odot}$. For the STD model, there is typically full fallback associated with the formation of BHs with masses of $\sim 7-8 M_{\odot}$, and no NK is applied. Even more dissimilar is the $v_{\mathrm{BH}, 0}$ distribution for the $\mathrm{NK}_{\mathrm{BE}}$ model, where $\mathrm{NKs}$ are proportional to the ratio of ejected mass to compact object mass ( $v_{\mathrm{NK}} \sim M_{\mathrm{ej}} / M_{\mathrm{BH}}$; Equation (3)) and typically, for $7.5 M_{\odot}$ BHs, are $\sim 130 \mathrm{~km} \mathrm{~s}^{-1}$.

\subsection{Bound Systems}

The number of BHs that are bound in binaries is between $5.7 \times 10^{4}$ and $2.1 \times 10^{7}$ (depending on the model, see Table 2) per MWEG. The fraction of BHs that reside in binaries for most of the tested models is $\lesssim 17 \%$. For models $\mathrm{NK}_{\mathrm{R}}$ and $\mathrm{NK}_{\mathrm{BE}}$, the fraction of $\mathrm{BHs}$ in binaries is $\lesssim 0.1 \%$, so the model of NKs highly influences the survival of $\mathrm{BH}$ binaries.

The maximum mass for a $\mathrm{BH}$ is $\sim 22 M_{\odot}$ (or $\sim 45$ for lower metallicity models). The binary with such a massive $\mathrm{BH}$ exists for $\sim 100 \mathrm{kyr}$ before it disrupts after the second SN. Afterward, these BHs become the most heavy dSBHs (Section 3.1.2).

Most of the binaries harboring BHs are wide, that is, formed without any interactions between stars. BHs reside predominantly in DCOs (Section 3.2.1). The rest are mostly accompanied by WDs (such binaries we treat as non-DCOs, i.e., BHBs; Section 3.2.2). A small fraction of BHBs could be observed during their MT phase, although this fraction is strongly dependent on the adopted SFH (Section 3.2.3).

\subsubsection{Double Compact Objects}

In this study, by DCOs we mean only those harboring at least one $\mathrm{BH}$ (i.e., $\mathrm{BH}+\mathrm{BH}$ and $\mathrm{BH}+\mathrm{NS}$ ), totally neglecting $\mathrm{NS}+\mathrm{NS}$ systems. $\mathrm{BH}+\mathrm{WD}$ systems are included in non-DCO binaries (BHBs; see Section 3.2.2). The fact that the majority of binaries harboring $\mathrm{BHs}$ are actually DCOs is a direct consequence of the adopted uniform distribution of mass ratios on ZAMS: if the primary is a $\mathrm{BH}$ progenitor $\left(M_{\mathrm{ZAMS}} \gtrsim\right.$ $\left.20 M_{\odot}\right)$, the most likely companions are NS and $\mathrm{BH}$ progenitors $\left(M_{\text {ZAMS }} \gtrsim 8 M_{\odot}\right)$. NSs generally receive higher NKs than BHs, and many potential $\mathrm{BH}+\mathrm{NS}$ binaries get disrupted during the NS formation (e.g., Dominik et al. 2012); therefore, $\mathrm{BH}+\mathrm{BH}$ systems typically dominate ( $95 \%-98 \%$; see Table 5) over $\mathrm{BH}+\mathrm{NS}$ binaries in most tested models. The exception are models with higher average $\mathrm{NKs}\left(\mathrm{NK}_{\mathrm{R}}\right.$ and $\mathrm{NK}_{\mathrm{BE}}$, where most of the DCOs are compact $\mathrm{BH}+\mathrm{NS}$ systems. Many groups studied the formation of DCOs harboring BHs over the years (e.g., Lipunov et al. 1997b; Nelemans et al. 2001; Belczynski et al. 2002b; Voss \& Tauris 2003; Dominik et al. 2012; Belczynski et al. 2016a, 2016b; Eldridge \& Stanway 2016; Stevenson et al. 2017). However, the main focus of those studies was the merger rates and properties of gravitational wave sources, so they performed no deeper analysis of wider DCOs. Here, we present the analysis of the entire DCO population as part of the total $\mathrm{BH}$ population. Therefore, our results may be applied not only to the study of merging DCOs but also to other phenomena, such as microlensing by DCOs.

In Table 5 we present detailed results for the main models. The number of BHs in DCOs is typically between $1.5 \times 10^{6}$ and $1.9 \times 10^{7}$ (note that $\mathrm{BH}+\mathrm{BH}$ counts as two $\mathrm{BHs}$ ), although for the $\mathrm{NK}_{\mathrm{R}}$ and $\mathrm{NK}_{\mathrm{BE}}$ models, the number drops to $5.1 \times 10^{3}$ and $2.5 \times 10^{2}$, respectively. The low number of DCOs in the models with higher average NKs is connected to the fact that most binaries with massive components that are potential progenitors of DCOs are wide (route $\mathcal{R}_{\mathrm{DCO}, 1}$; see below) and thus are easily disrupted by even moderate NKs.

Two main evolutionary routes lead to the formation of DCOs harboring BHs (Table 3). The main feature that distinguishs these routes is the initial separation. It is connected with the lack $\left(\mathcal{R}_{\mathrm{DCO}, 1}\right)$ or presence $\left(\mathcal{R}_{\mathrm{DCO}, 2}\right)$ of the pre-DCO formation interactions (MT and $\mathrm{CE}$ ), which results in the formation of wide $\left(a \gtrsim 10^{4}\right)$ or close $\left(a \lesssim 10^{3} R_{\odot}\right)$ DCOs, respectively (see Figure 6). Among close DCOs (mainly route $\mathcal{R}_{\mathrm{DCO}, 2}$ ) are potential DCO merger progenitors (e.g., Abbott et al. 2016a), and their number and relative fraction strongly depend on metallicity and NKs, as previously noted by Chruslinska et al. (2019).

Route $\mathcal{R}_{\mathrm{DCO}, 1}$ is the main formation route of DCOs if the standard NK model is concerned. In this formation channel (Figure 7, upper plot), none of the strong interactions are present. In a typical situation, the stars on the ZAMS are not very massive $\left(\sim 25\right.$ and $\left.\sim 22 M_{\odot}\right)$ and the separation is large $\left(\sim 12,300 R_{\odot}\right)$. After about $7.7 \mathrm{Myr}$, the primary explodes and forms a $\sim 7.5 M_{\odot} \mathrm{BH}$. In an additional $\sim 1 \mathrm{Myr}$, the secondary forms a $7.5 \mathrm{Myr} \mathrm{BH}$ in an $\mathrm{SN}$ explosion. The final separation is $\sim 37,500 R_{\odot}$. 
Table 5

Double Compact Objects Containing Black Holes

\begin{tabular}{lccccccrrrrr}
\hline \hline Model & $N_{\text {BH,DCO }}$ & \multicolumn{2}{c}{ BH+BH } & \multicolumn{2}{c}{$\mathcal{R}_{\mathrm{DCO}, 1}$} & \multicolumn{2}{c}{$\mathcal{R}_{\mathrm{DCO}, 2}$} & \multicolumn{2}{c}{$a\left[R_{\odot}\right]<10^{3} R_{\odot}$} & $t_{\text {merge }}<10 \mathrm{Gyr}$ \\
\hline STD & $5.1 \times 10^{6}$ & $4.9 \times 10^{6}$ & $(95.9 \%)$ & $2.4 \times 10^{6}$ & $(90.1 \%)$ & $1.6 \times 10^{4}$ & $(0.6 \%)$ & $2.5 \times 10^{4}$ & $(0.9 \%)$ & $1.7 \times 10^{4}$ & $(0.6 \%)$ \\
mid-Z & $1.2 \times 10^{7}$ & $1.2 \times 10^{7}$ & $(96.6 \%)$ & $2.6 \times 10^{6}$ & $(42.6 \%)$ & $4.0 \times 10^{5}$ & $(3.4 \%)$ & $1.7 \times 10^{6}$ & $(27.3 \%)$ & $8.1 \times 10^{5}$ & $(13.0 \%)$ \\
low-Z & $1.9 \times 10^{7}$ & $1.9 \times 10^{7}$ & $(97.5 \%)$ & $5.7 \times 10^{6}$ & $(57.8 \%)$ & $1.8 \times 10^{6}$ & $(18.1 \%)$ & $2.7 \times 10^{6}$ & $(27.0 \%)$ & $1.9 \times 10^{6}$ & $(19.0 \%)$ \\
SS0 & $6.9 \times 10^{6}$ & $6.6 \times 10^{6}$ & $(95.6 \%)$ & $3.4 \times 10^{6}$ & $(93.1 \%)$ & $2.4 \times 10^{4}$ & $(0.7 \%)$ & $3.0 \times 10^{4}$ & $(0.8 \%)$ & $2.3 \times 10^{4}$ & $(0.6 \%)$ \\
NK $_{\mathrm{R}}$ & $1.1 \times 10^{4}$ & $5.1 \times 10^{3}$ & $(45.5 \%)$ & $8.8 \times 10^{2}$ & $(10.3 \%)$ & $1.6 \times 10^{3}$ & $(19.1 \%)$ & $6.8 \times 10^{3}$ & $(79.4 \%)$ & $3.2 \times 10^{3}$ & $(36.8 \%)$ \\
NK $_{\text {BE }}$ & $2.7 \times 10^{3}$ & $2.5 \times 10^{2}$ & $(9.5 \%)$ & $\ldots$ & $\ldots$ & $2.5 \times 10^{3}$ & $(100 \%)$ & $1.3 \times 10^{2}$ & $(5.0 \%)$ & \\
flat IMF & $1.3 \times 10^{7}$ & $1.3 \times 10^{7}$ & $(96.6 \%)$ & $6.2 \times 10^{6}$ & $(90.6 \%)$ & $3.8 \times 10^{4}$ & $(0.6 \%)$ & $6.0 \times 10^{4}$ & $(0.9 \%)$ & $3.8 \times 10^{4}$ & $(0.6 \%)$ \\
steep IMF & $1.6 \times 10^{6}$ & $1.5 \times 10^{6}$ & $(95.3 \%)$ & $7.7 \times 10^{5}$ & $(90.7 \%)$ & $3.7 \times 10^{3}$ & $(0.4 \%)$ & $5.7 \times 10^{3}$ & $(0.7 \%)$ & $3.7 \times 10^{3}$ & $(0.4 \%)$ \\
\hline
\end{tabular}

Note. Results for double compact objects harboring BHs (i.e., $\mathrm{BH}+\mathrm{BH}$ and $\mathrm{BH}+\mathrm{NS}$ systems): $N_{\mathrm{BH}, \mathrm{DCO}}$, number of $\mathrm{BHs}$ in double compact objects; $\mathrm{BH}+\mathrm{BH}$, number of BHs in double $\mathrm{BH}$ systems (the rest reside in $\mathrm{BH}+\mathrm{NS}$ systems); $\mathcal{R}_{\mathrm{DCO}, 1}$ and $\mathcal{R}_{\mathrm{DCO}, 2}$, number of BHs formed through specific evolutionary routes; $a\left[R_{\odot}\right]<10^{3} R_{\odot}$, number of BHs residing in close DCOs, that is, systems with separations lower than $10^{3} R_{\odot} ; t_{\text {merge }}<10$ Gyr, number of BHs in systems with time to merger after the second supernova smaller than $10 \mathrm{Gyr}$.

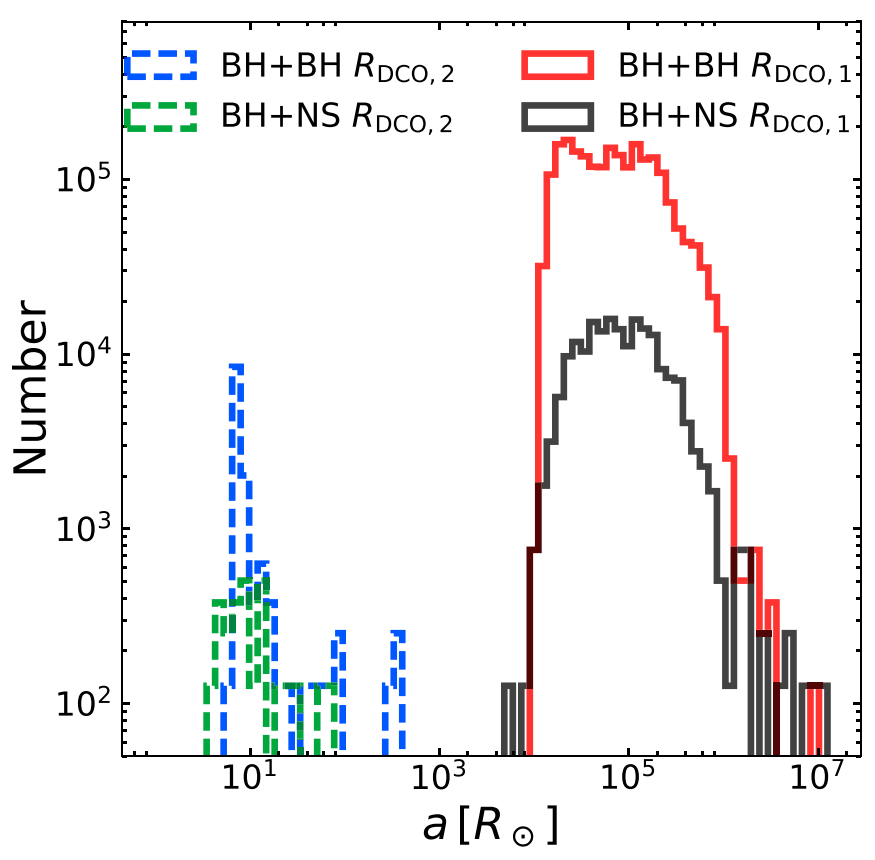

Figure 6. Distribution of separations of double compact objects at the moment of their formation for the STD model. Presented are distributions for $\mathrm{BH}+\mathrm{BH}$ and $\mathrm{BH}+\mathrm{NS}$ binaries and two main evolutionary routes $\mathcal{R}_{\mathrm{DCO}, 1}$ and $\mathcal{R}_{\mathrm{DCO}, 2}$. A gap at $\sim 10^{3}-10^{4} R_{\odot}$ separates double compact objects originating from different evolutionary channels. Large separations are a property of systems that were formed without any binary interactions $\left(\mathcal{R}_{\mathrm{DCO}, 1}\right.$ route). Double compact objects formed in the $\mathcal{R}_{\mathrm{DCO}, 2}$ route have a smaller separation $\left(a \lesssim 10^{3} R_{\odot}\right)$, which is a direct consequence of a $\mathrm{CE}$ phase during the formation process.

The other formation route $\left(\mathcal{R}_{\mathrm{DCO}, 2}\right)$ is responsible for a much smaller fraction of DCOs $(\lesssim 21 \%)$, but systems that form through this route have much shorter separations and, thus, may merge after less than 10 Gyr due to the emission of gravitational waves. In a typical case, the binary is massive on ZAMS $\left(M_{\mathrm{ZAMS}, \mathrm{a}} \approx 57 M_{\odot}\right.$ and $M_{\mathrm{ZAMS}, \mathrm{b}} \approx 38 M_{\odot}$ ), and the separation is small $\left(a_{\mathrm{ZAMS}} \approx 600 R_{\odot}\right)$. The primary evolves fast and after $\sim 4.1$ Myr fills its Roche lobe while expanding on the HG. The MT commences and results in the loss of nearly the entire hydrogen envelope by the primary, which shortly after becomes a $\sim 16 M_{\odot}$ HeS. Half of the primary's envelope is accreted by the secondary, which grows to $\sim 49 M_{\odot}$ while still on its MS. After an additional $\sim 600 \mathrm{kyr}$, the primary forms a $\sim 8 M_{\odot} \mathrm{BH}$ in an $\mathrm{SN}$ explosion. The secondary starts to

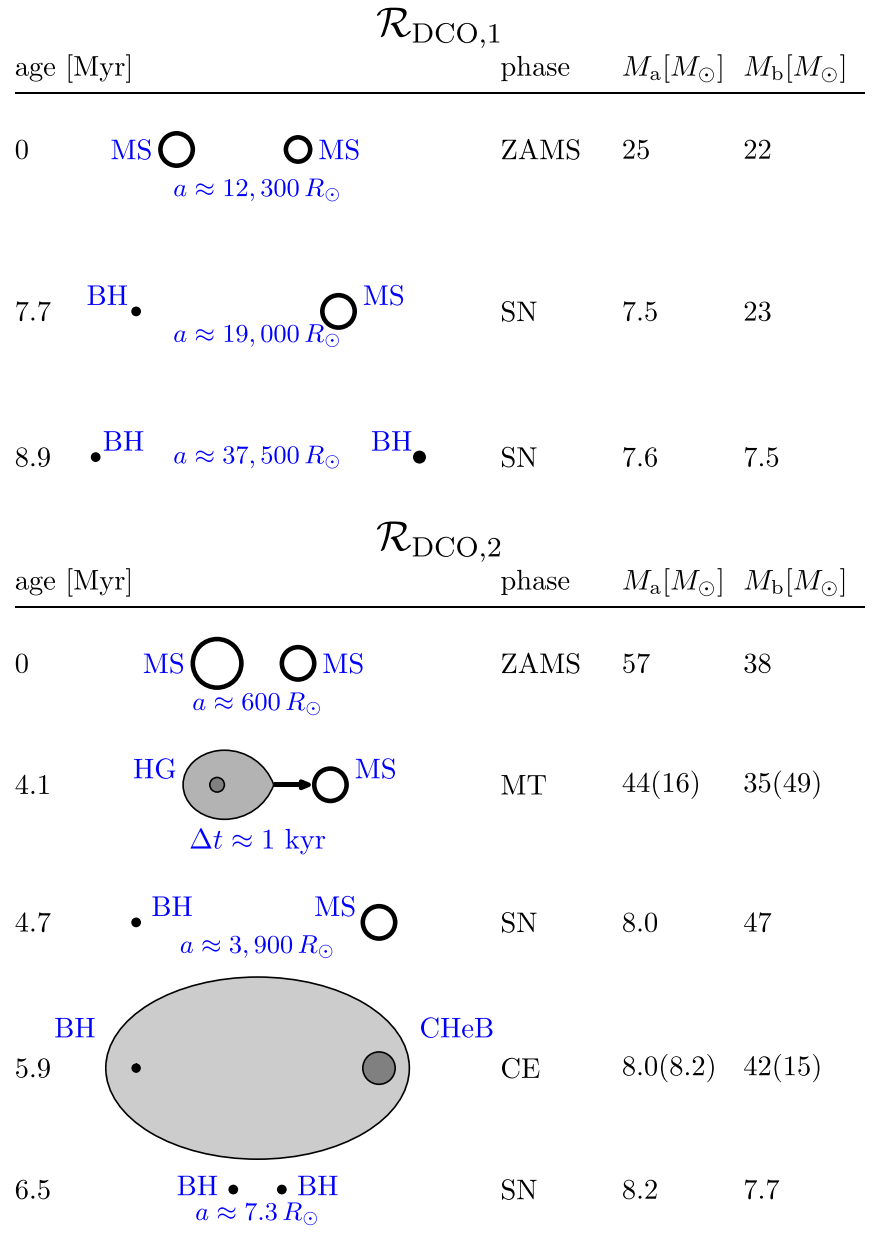

Figure 7. Main evolutionary phases leading to the formation of DCOs harboring BHs. Route $\mathcal{R}_{\mathrm{DCO}, 1}$ is presented on the upper plot, and route $\mathcal{R}_{\mathrm{DCO}, 2}$ on the lower one. For explanations of the majority of abbreviations, see Figure 2. Additionally: $\mathrm{CE}$, common envelope; $\mathrm{CHeB}$, core helium burning.

expand after leaving the MS, and while being a $\mathrm{CHeB}$ star, it fills its Roche lobe and a CE commences. As a result, the separation shrinks from $\sim 4000 R_{\odot}$ to about $5.2 R_{\odot}$, and the secondary loses its entire envelope and becomes a $\sim 15 M_{\odot}$ HeS. A second $\mathrm{SN}$ occurs $\sim 600 \mathrm{kyr}$ later and a $\sim 7.7 M_{\odot}$ BH forms. The $\mathrm{NK}$ is not very strong, so the separation remains small $\left(\sim 7.3 R_{\odot}\right)$. Such a binary is estimated to merge after $\sim 420 \mathrm{Myr}$. This channel was previously 
thoroughly analyzed in the context of DCO mergers by, for example, Belczynski et al. (2002b), Dominik et al. (2012), Belczynski et al. (2016b), Woosley (2016), and Kruckow et al. (2018).

$\mathrm{BH}+\mathrm{NS}$ formation through $\mathcal{R}_{\mathrm{DCO}, 1}$ and $\mathcal{R}_{\mathrm{DCO}, 2}$ routes is qualitatively similar to the formation of $\mathrm{BH}+\mathrm{BH}$ systems, although the ZAMS secondary masses are significantly lower $\left(\sim 3 \times\right.$ for $\mathcal{R}_{\mathrm{DCO}, 1}$ and $\sim 1.5 \times$ for $\left.\mathcal{R}_{\mathrm{DCO}, 2}\right)$. Additionally, the progenitors are more easily disrupted during the second $\mathrm{SN}$ and have a smaller chance of $\mathrm{CE}$ survival, due to a more extreme mass ratio. This results in a significantly lower fraction of $\mathrm{BH}$ $+\mathrm{NS}$ systems among DCOs than $\mathrm{BH}+\mathrm{BH}$ systems, in spite of a higher abundance of potential progenitors in initial (ZAMS) populations. ${ }^{6}$ There are typically (except $\mathrm{NK}_{\mathrm{R}}$ and $\mathrm{NK}_{\mathrm{BE}}$ models) $\gtrsim 20$ times more $\mathrm{BHs}$ in $\mathrm{BH}+\mathrm{BH}$ binaries than in $\mathrm{BH}$ $+\mathrm{NS}$. We observe no significant difference between the shapes of $\mathrm{BH}+\mathrm{BH}$ and $\mathrm{BH}+\mathrm{NS}$ distributions of orbital separations at the moment of of DCO formation (Figure 6).

We have found that the number of BHs in DCOs is 3-4 times higher in lower metallicity environments than in the STD model. The number of wide DCOs $\left(\mathcal{R}_{\mathrm{DCO}, 1}\right)$ is only slightly affected by metallicity (by a factor of $\lesssim 2.5$ ), whereas the number of close DCOs $\left(\mathcal{R}_{\mathrm{DCO}, 2}\right)$ is significantly affected (by more than two orders of magnitude). The latter is an effect of higher BH masses (typically $\sim 15$ ) in low-metallicity environments in comparison to $Z_{\odot}$ environments (typically $\sim 7-8 M_{\odot}$ ). Heavier BHs in the standard NK model usually obtain lower NKs due to fallback, or no NK when formed in a direct collapse. Therefore, DCOs in lower metallicity environments, where BHs form with higher masses, are less frequently disrupted (e.g., Belczynski et al. 2010b).

A significantly different situation concerns the relation for close systems $\left(\mathcal{R}_{\mathrm{DCO}, 2}\right)$, which in lower metallicity environments are about two orders of magnitude more numerous than in the STD model. This results from an interplay between mass loss in the stellar wind and stellar expansion (Figure 4). The former is proportional to metallicity and leads to orbital widening, due to mass loss from the system. On the other hand, solar-metallicity stars do not expand as significantly as those in

\footnotetext{
6 The ratio of potential $\mathrm{BH}+\mathrm{NS}$ progenitors to potential $\mathrm{BH}+\mathrm{BH}$ progenitors (i.e., binaries with component masses high enough to form an $\mathrm{NS}$, or $\mathrm{BH}$ in single-star evolution) may be calculated as$$
f=\frac{\int_{M_{\mathrm{NS}, \mathrm{ZAMS}, \max }}^{150 M_{\odot}} P_{\mathrm{BH}+\mathrm{NS}}\left(M_{1}\right) \operatorname{IMF}\left(M_{1}\right) d M_{1}}{\int_{M_{\mathrm{NS}, \mathrm{ZAMS}, \max }}^{150 M_{\odot}} P_{\mathrm{BH}+\mathrm{BH}}\left(M_{1}\right) \operatorname{IMF}\left(M_{1}\right) d M_{1}},
$$

where $P_{\mathrm{BH}+\mathrm{NS} / \mathrm{BH}+\mathrm{BH}}\left(M_{1}\right)$ is the probability that a companion of a $\mathrm{BH}$ progenitor with mass $M_{1}$ is an NS/BH progenitor. Assuming a flat mass ratio distribution for companions $\left(P(q)=1 /\left(M_{1}-M_{\mathrm{NS}, \mathrm{ZAMS} \text {, min }}\right)\right)$, we have

$$
P_{\mathrm{BH}+\mathrm{NS}}\left(M_{1}\right)=\frac{M_{\mathrm{NS}, \mathrm{ZAMS}, \max }-M_{\mathrm{NS}, \mathrm{ZAMS}, \text { min }}}{M_{1}-M_{\mathrm{NS}, \mathrm{ZAMS}, \min }}
$$

and$$
P_{\mathrm{BH}+\mathrm{BH}}\left(M_{1}\right)=\frac{M_{1}-M_{\mathrm{NS}, \mathrm{ZAMS}, \mathrm{max}}}{M_{1}-M_{\mathrm{NS}, \mathrm{ZAMS}, \min }},
$$

where $M_{\mathrm{NS}, \mathrm{ZAMS} \text {, } \min / \max }=8 / 22 M_{\odot}$ are the minimal $/$ maximal ZAMS masses for an NS progenitor (assuming no interactions with the companion) for solar metallicity $\left(Z_{\odot}\right)$. IMF $\left(M_{1}\right) \propto M_{1}^{\Gamma}$ is the IMF. $150 M_{\odot}$ is the upper limit for the ZAMS mass adopted in our calculations. This assumes $\Gamma=-2.3$ and solar metallicity (STD model) $f \approx 1.1$, meaning more $\mathrm{BH}+\mathrm{NS}$ than $\mathrm{BH}+\mathrm{BH}$ progenitors in initial populations. This value is slightly affected by metallicity, as $Z=1 \% Z_{\odot}$ (low- $Z$ model; where $\left.M_{\mathrm{NS}, Z \text { AMS, } \max } \approx 19 M_{\odot}\right)$ gives $f \approx 1$, and significantly affected by the steepness of the IMF, as $\Gamma=-1.9$ (flat IMF model) gives $f \approx 0.9$, i.e., more $\mathrm{BH}+\mathrm{BH}$ progenitors.
}
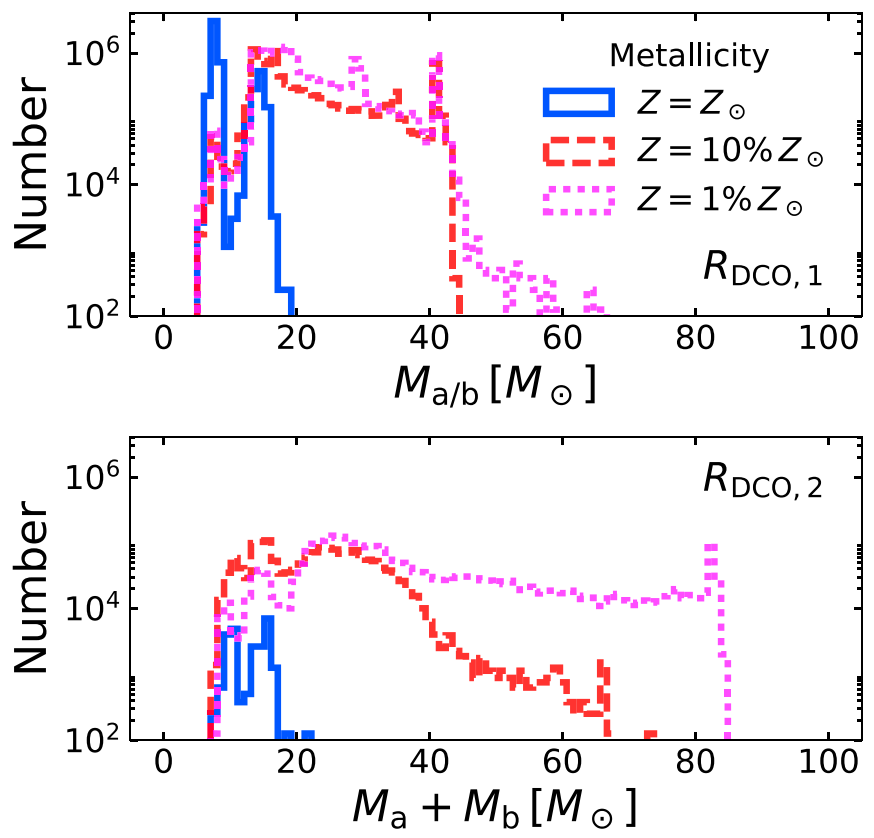

Figure 8. Distribution of component masses of wide double compact objects ( $a>10^{3} R_{\odot}$; route $\mathcal{R}_{\mathrm{DCO}, 1}$; upper plot) and total binary masses for close double compact objects $\left(a \leqslant 10^{3} R_{\odot}\right.$; route $\mathcal{R}_{\mathrm{DCO}, 2}$; lower plot) scaled for MWEG. Different metallicities are presented: $Z=Z_{\odot}$ (STD model), $Z=10 \% Z_{\odot}$ (mid- $Z$ model), and $Z=1 \% Z_{\odot}$ (low- $Z$ model). For wide $\mathrm{BH}$ +NS systems (upper plot), only BH masses are presented.

the mid- $Z$ model. This means that massive binaries in a highmetallicity environment (e.g., STD model) more frequently have separations too large to go through a CE phase, which is an essential step in the $\mathcal{R}_{\mathrm{DCO}, 2}$ route. Consequently, lower metallicity models have more close DCOs than high-metallicity ones. A similar result was recently obtained by Spera et al. (2019) with the use of the SEVN population synthesis code.

Distributions of component masses are shown in Figure 8. For the $\mathcal{R}_{\mathrm{DCO}, 1}$ formation channel (wide DCOs), distributions for primaries and secondaries are joined together, except $\mathrm{BH}$ $+\mathrm{NS}$ systems, for which only $\mathrm{BH}$ masses are shown. The results resemble those for $\mathrm{BHs}$ from disrupted binaries (Figure 3). For the $\mathcal{R}_{\mathrm{DCO}, 2}$ channel (close DCOs), the total mass of a binary is provided. Such information is more important from the point of view of microlensing surveys, because a close binary will most probably act as a single lens. For lower metallicities, the total mass may reach $\sim 70-80 M_{\odot}$, which matches the current range of observed mass in $\mathrm{BH}+\mathrm{BH}$ mergers (The LIGO Scientific Collaboration et al. 2019; see also Belczynski et al. 2016b).

Within our models, up to $\sim 15 \%$ of BHs are found in DCOs (Table 2), which are predominantly wide (Voss \& Tauris 2003). For the standard NK model, up to $19 \%$ of them have merger times $\left(t_{\text {merge }}\right)$ smaller than $10 \mathrm{Gyr}$, which mainly depends on metallicity (see Table 5; merging $\mathrm{BH}+\mathrm{BH}$ systems form much more efficiently at low Z, e.g., Dominik et al. 2013; Giacobbo \& Mapelli 2018; Klencki et al. 2018; Chruslinska et al. 2019). In the $\mathrm{NK}_{\mathrm{R}}$ and $\mathrm{NK}_{\mathrm{BE}}$ models, although the fraction of merging systems may be much higher $\left(\sim 37 \%\right.$ for $\mathrm{NK}_{\mathrm{R}}$ model), the number of these systems is significantly smaller. This is a consequence of higher average NKs $\left(\sim 125-130 \mathrm{~km} \mathrm{~s}^{-1}\right.$ in contrast to $\sim 10 \mathrm{~km} \mathrm{~s}^{-1}$ in the STD model), which frequently disrupt wide binaries. 


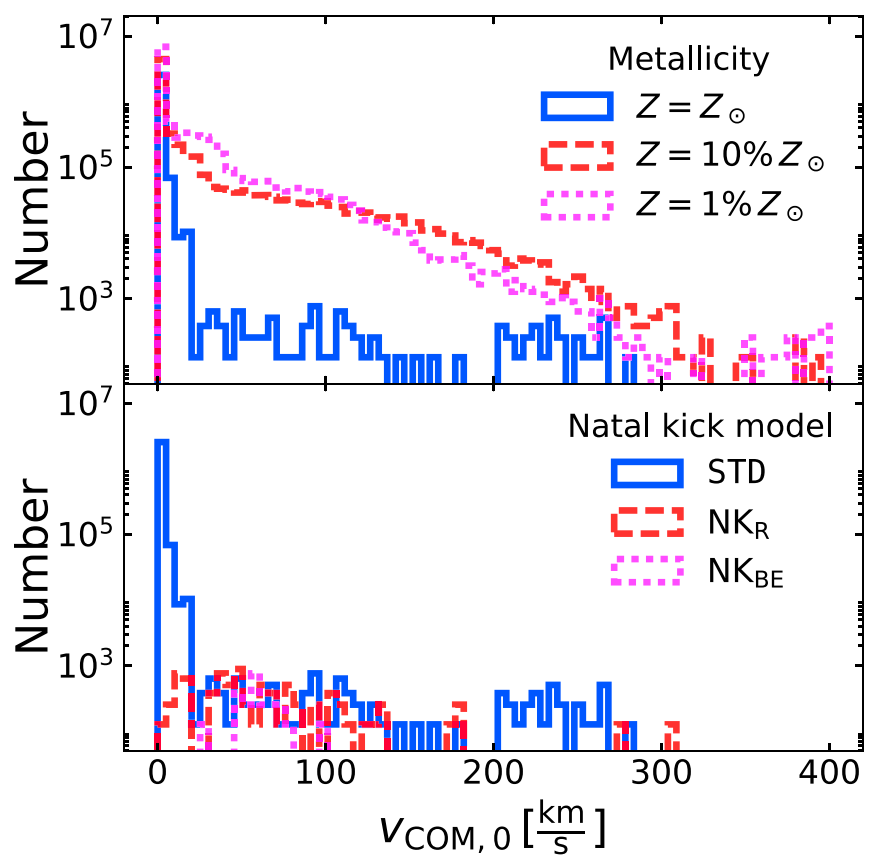

Figure 9. Distribution of the initial center-of-mass velocities of double compact objects just after the formation of the second compact object ( $\left.v_{\mathrm{COM}, 0}\right)$. Note that the motion in the gravitational potential is not included here. The upper plot compares distributions for different metallicities, whereas the lower one compares different NK models.

Typical center-of-mass initial velocities ( $v_{\mathrm{COM}, 0}$; i.e., binary velocities just after the second $\mathrm{SN}$ not including velocity resulting from the motion in a gravitational potential) are below $\sim 20 \mathrm{~km} \mathrm{~s}^{-1}$ (Figure 9). Only less than $15 \%$ of DCOs (mainly compact; route $\mathcal{R}_{\mathrm{DCO}, 2}$ ) possess velocities exceeding $20 \mathrm{~km} \mathrm{~s}^{-1}$. Therefore, the vast majority of the DCOs remain in the vicinity of their birthplaces. The highest velocities $\left(v_{\mathrm{COM}, 0} \gtrsim 200 \mathrm{~km} \mathrm{~s}^{-1}\right)$ are obtained by $\mathrm{BH}+\mathrm{NS}$ systems, whereas systems with lower velocities are dominated by $\mathrm{BH}$ $+\mathrm{BH}$ binaries, which results from the typically higher NKs obtained by NSs than BHs. Typical velocities of merging systems $\left(t_{\text {merge }}<10 \mathrm{Gyr}\right)$ are below $\sim 50 \mathrm{~km} \mathrm{~s}^{-1}$ for the STD model, so most of the mergers are predicted to occur in the vicinity of the formation places (e.g., Perna et al. 2018). However, some systems may also obtain high velocities $\left(\gtrsim 200 \mathrm{~km} \mathrm{~s}^{-1}\right)$. These are mainly $\mathrm{BH}+\mathrm{NS}$ systems, which we predict to merge away from their birth environments. For $\mathrm{NK}_{\mathrm{R}}$ and $\mathrm{NK}_{\mathrm{BE}}$ models, DCOs obtain typically intermediate velocities $\left(\sim 50-130 \mathrm{~km} \mathrm{~s}^{-1}\right.$; mainly route $\mathcal{R}_{\mathrm{DCO}, 2}$; typically, $t_{\text {merge }} \lesssim 10 \mathrm{Gyr}$ ), although some systems (mainly $\mathrm{BH}+\mathrm{NS}$ ) may have velocities as high as $\sim 400 \mathrm{~km} \mathrm{~s}^{-1}$.

\subsubsection{Wide Non-DCO Binaries}

Throughout the paper, we use the term wide BHB to refer to systems harboring a $\mathrm{BH}$ with a noncompact companion and experiencing no strong interaction during their entire evolution (the separation between the stars is large, and the stars never fill their Roche lobes). In this section, we focus on wide BHBs, excluding wide DCOs, which were discussed in the previous section.

Table 6 summarizes our results for the main models. The typical number of wide BHBs is between $5.5 \times 10^{5}$ and $3.3 \times 10^{6} M_{\odot}$. Only for models with higher average NKs $\left(\mathrm{NK}_{\mathrm{R}}\right.$ and $\left.\mathrm{NK}_{\mathrm{BE}}\right)$ is the predicted number of such binaries much lower $\left(3.1 \times 10^{3}\right.$ and $1.0 \times 10^{-4}$, respectively). The probability for a binary to obtain a low NK $\left(\lesssim 20 \mathrm{~km} \mathrm{~s}^{-1}\right)$ in $\mathrm{NK}_{\mathrm{R}}$ and $\mathrm{NK}_{\mathrm{BE}}$ models is much lower than in the STD model (see Figure 5), and typically NKs are $\gtrsim 100 \mathrm{~km} \mathrm{~s}^{-1}$. Therefore, in these models, wide binaries are more easily disrupted during the formation of a $\mathrm{BH}$. In contrast, the standard $\mathrm{NK}$ model (Hobbs et al. 2005) predicts mostly low or negligible NKs for BHs, which allows for more frequent survival of wide binaries.

The number of wide BHBs is higher in the STD model than in the mid- $Z$ and low $-Z$ models mainly because of the more significant orbital expansion resulting from mass loss in stellar wind, which is stronger for higher metallicities. Additionally, stellar expansion is higher in the mid- $Z$ model, thus further increasing the chance of interactions (Figure 4). In the low- $Z$ model, stellar expansion is smaller than in the mid- $Z$ model, therefore resulting in a higher number of wide BHBs.

$\mathrm{BH}$ companions in wide systems are predominantly $\mathrm{CO}$ WDs (74\%-89\%). WDs, being the final evolutionary stage of low-mass $\left(\lesssim 8 M_{\odot}\right)$ star evolution, are simultaneously the longest evolutionary stage of the evolution of stars with initial masses $2 \lesssim M \lesssim 8 M_{\odot}$ during the Hubble time. For comparison, low-mass stars $\left(M \lesssim 1 M_{\odot}\right)$ spend most of this time on the MS, whereas heavier stars $\left(M \gtrsim 8 M_{\odot}\right)$ quickly $(\lesssim 50 \mathrm{Myr})$ end their evolution and form an NS or a BH. The typical separations of wide BHBs are $a \gtrsim 5000$.

In an example evolution of a typical wide $\mathrm{BHB}$, the progenitor system on ZAMS is composed of a $\sim 25 M_{\odot}$ primary and a $6.0 M_{\odot}$ companion (see Figure 10). The initial separation is large $\left(\sim 16,000 R_{\odot}\right)$, which precludes any interactions. The separation further increases with wind mass loss from the stars and just before the $\mathrm{SN}$ reaches $\sim 34,000 R_{\odot}$. The primary, being heavier, evolves faster and in $\sim 7.8 \mathrm{Myr}$ becomes a BH. The NK and the Blaauw kick are crucial at that point. Only a small total kick will allow for survival of the binary. The secondary is still on its MS for another $\sim 60 \mathrm{Myr}$. At the age of about $76 \mathrm{Myr}$, a CO WD forms. The final separation may reach $56,000 R_{\odot}$.

The mass distribution of BHs in wide BHBs (Figure 11) resembles that for $\mathrm{dSBHs}$ as most $\mathrm{dSBH}$ are not interacting significantly prior to disruption and thus frequently originate from wide BHBs. Two distributions differ in the presence (dSBHs) or lack (wide BHBs) of the high-mass peak ( 22 $\left.M_{\odot}\right)$ for the STD model. Also, the high-mass tail (above $\gtrsim 15 M_{\odot}$ for the STD model and $\gtrsim 40 M_{\odot}$ for mid- $Z$ and low$Z$ models), which originates from wind accretion by a BH or its progenitor, in the case of noninteracting binaries is less pronounced than in distributions for interacting binaries. The maximal $\mathrm{BH}$ masses are $\sim 16 M_{\odot}$ for the STD model and $\sim 46 M_{\odot}$ in lower metallicities. The distribution of companion masses is dominated by WDs (carbon-oxygen and oxygenneon), which form a characteristic peak between $\sim 1 M_{\odot}$ and the Chandrasekhar mass $\left(M_{\mathrm{Ch}}=1.44 M_{\odot}\right)$. The long tail on this distribution is mainly composed of MS stars, which may have masses up to $\sim 80 M_{\odot}$ for solar metallicity or $\gtrsim 100 M_{\odot}$ for lower metallicity models. The massive companions $\left(M \gtrsim 8 M_{\odot}\right)$ are going to explode in an SN explosion, which may disrupt the BHBs (either through an NK or a Blaauw kick) populating $\mathrm{dSBHs}$ or form wide DCOs (discussed in Section 3.2.1).

Although in rare cases the initial center-of-mass velocity $\left(v_{\mathrm{COM}, 0}\right)$ of wide BHBs may reach $\sim 80 \mathrm{~km} \mathrm{~s}^{-1}$, typically ( $\gtrsim 95 \%$ of cases) they are negligible $\left(\lesssim 10 \mathrm{~km} \mathrm{~s}^{-1}\right)$. Because orbital velocities are typically also small $\left(\lesssim 20 \mathrm{~km} \mathrm{~s}^{-1}\right)$, the 
Table 6

Wide and Mass-transferring Binaries

\begin{tabular}{|c|c|c|c|c|c|c|}
\hline \multirow{2}{*}{ Model } & \multicolumn{3}{|c|}{ Wide BHB } & \multicolumn{3}{|c|}{ МТВНВ } \\
\hline & $N$ & $a\left[R_{\odot}\right]$ & Typ. Comp. & $N$ & $a\left[R_{\odot}\right]$ & Typ. Comp. \\
\hline STD & $1.5 \times 10^{6}$ & $\gtrsim 10,000$ & CO WD $(86 \%)$ & $8.5 \times 10^{1}$ & $\lesssim 115$ & MS (75\%) \\
\hline low-Z & $1.2 \times 10^{6}$ & $\gtrsim 5,800$ & CO WD $(85 \%)$ & $4.4 \times 10^{2}$ & $\underset{\sim}{\sim} 84$ & MS $(91 \%)$ \\
\hline SSO & $3.3 \times 10^{6}$ & $\gtrsim 8,300$ & CO WD $(74 \%)$ & $5.7 \times 10^{2}$ & $\lesssim 108$ & MS (73\%) \\
\hline $\mathrm{NK}_{\mathrm{R}}$ & $3.1 \times 10^{3}$ & $\gtrsim 4,800$ & CO WD (89\%) & $1.7 \times 10^{2}$ & $\lesssim 90$ & MS (86\%) \\
\hline $\mathrm{NK}_{\mathrm{BE}}$ & $1.0 \times 10^{-4}$ & $\gtrsim 14,000$ & $\mathrm{CHeB}(100 \%)$ & $1.8 \times 10^{2}$ & $\lesssim 75$ & MS $(82 \%)$ \\
\hline steep IMF & $5.5 \times 10^{5}$ & $\gtrsim 10,000$ & CO WD $(86 \%)$ & $3.2 \times 10^{1}$ & $\lesssim 116$ & MS (70\%) \\
\hline
\end{tabular}

Note. Results for wide and mass-transferring binaries with one of the components being a BH. $N$, number of binaries (also number of BHs); $a$, typical separation; Typ. comp., typical evolutionary type of the companion; MS, main sequence; $\mathrm{CHeB}$, core helium burning; $\mathrm{CO} \mathrm{WD}$, carbon-oxygen white dwarf.

\begin{tabular}{|c|c|c|c|c|c|c|}
\hline \multicolumn{4}{|c|}{ age [Myr] } & phase & $M_{\mathrm{a}}\left[M_{\odot}\right]$ & $M_{\mathrm{b}}\left[M_{\odot}\right.$ \\
\hline 0 & MS & 0 & MS & ZAMS & 25 & 6.0 \\
\hline 7.8 & $\mathrm{BH} \bullet$ & $a \approx 34,000 R_{\odot}$ & $\mathbf{o}^{\mathrm{MS}}$ & $\mathrm{SN}$ & 7.5 & 6.1 \\
\hline 76 & H & $a \approx 56,000 R_{\odot}$ & $\mathrm{CO}$ & WD form & 7.6 & 1.2 \\
\hline
\end{tabular}

Figure 10. Evolution toward the formation of a typical wide binary containing a BH (see Section 3.2.2). For descriptions of typical abbreviations and parameters, see Figure 2. Additionally: WD form, formation of a white dwarf; CO WD, carbon-oxygen white dwarf.
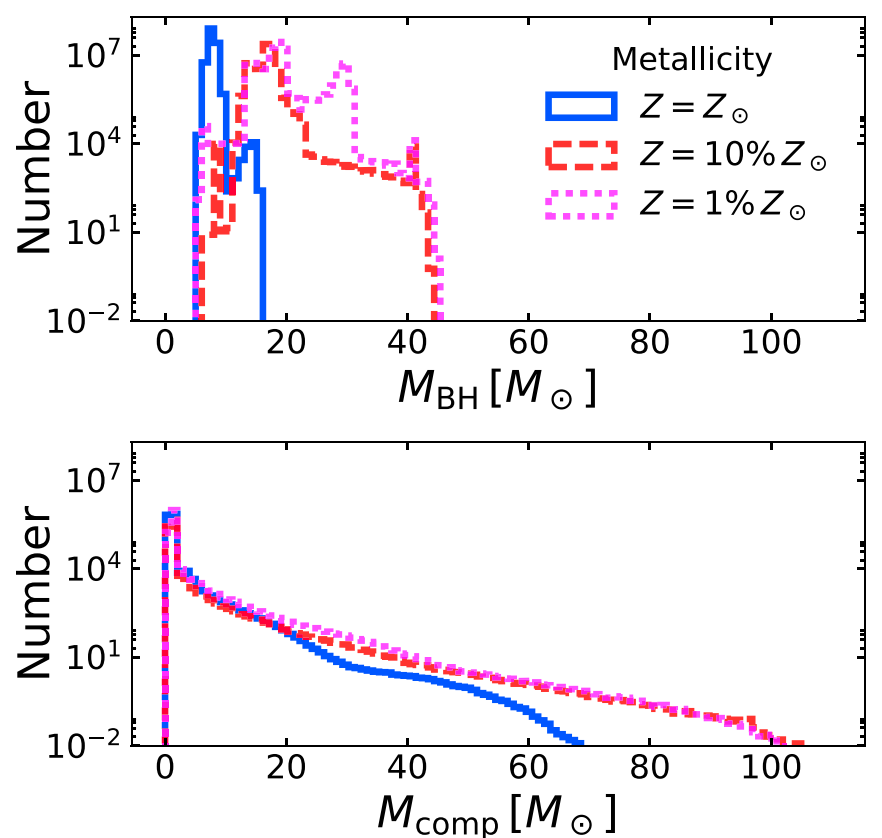

Figure 11. Distribution of $\mathrm{BH}$ ( $M_{\mathrm{BH}}$; upper plot) and companion ( $M_{\text {comp }}$; lower plot) masses for wide BHBs. Results for three metallicities are presented: $Z=Z_{\odot}$ (STD model), $Z=10 \% Z_{\odot}$ (mid- $Z$ model), and $Z=1 \% Z_{\odot}$ (low- $Z$ model). The peak in the companion's mass distribution is composed of WDs, whereas the tail is mostly formed of MS stars.

binaries rarely leave their birthplaces if evolved in isolation. We note that in a realistic situation a wide BHB is frequently influenced by flyby encounters (e.g., Klencki et al. 2017).

\subsubsection{Mass-transferring Binaries with BH Accretors}

Mass-transferring BHBs (MTBHB) have different evolutionary routes and properties than the typical BHBs, which are dominated by noninteracting systems (wide BHBs; Section 3.2.2). Although we note that $\mathrm{BHs}$ were detected also in high-mass XRBs where MT rates high enough to fuel an accretion disk may occur also through a companion's stellar wind (e.g., Ruhlen et al. 2011), in this study only RLOF systems were included.

For any adopted SFH, the fraction of BHs accreting mass from their companions through RLOF is small. However, this small fraction is more a result of the brevity of the MT phase in comparison to the evolutionary timescales than the rarity of BHBs that are close enough to commence RLOF. Here, for the sake of presentation, we assumed constant star formation for the last 10 Gyr. In such a case, only $<1 \%$ (see Table 6 ) of all BHBs contain a donor that is filling its Roche lobe and transferring mass onto a BH. MTBHBs can be perceptible as an XRB (e.g., Tauris \& van den Heuvel 2006). We found that, in our models, $2 \%-28 \%$ of BHs have gone through an MT phase that lasts typically $\lesssim 40 \mathrm{Myr}$. A $\mathrm{BH}$ accretes on average $0.7-3.1 M_{\odot}$ in that time.

Our simple model predicts 32-4200 MTBHBs per MWEG. The number is the highest in lower metallicities $\left(4.4 \times 10^{2}\right.$ and $4.2 \times 10^{3}$ systems for low- $Z$ and mid- $Z$ models, respectively), where it is easier to start RLOF MT (Linden et al. 2010). Also, in the SS0 model, we see an $\sim 8$ times higher predicted number of MTBHBs than in the STD model. This is a result of assuming in this model a thermal distribution of eccentricities $(P(e) \sim e)$, which significantly increases the average initial eccentricity, thus lowering the initial periastron distance, which makes RLOF easier.

There are two main evolutionary routes (Table 3) typical for all models (comprising $79 \%$ to $99 \%$ of all MTBHBs). Both channels differ mainly in the mass of an accreting BH. In the $\mathcal{R}_{\mathrm{MTBHB}, 1}$ route, typical BH masses are above $\sim 5 M_{\odot}$, whereas in the $\mathcal{R}_{\mathrm{MTBHB}, 2}$ route, they are around $\sim 3 M_{\odot}$. This distinctness stems from the different $\mathrm{BH}$ formation mechanisms. In $\mathcal{R}_{\mathrm{MTBHB}, 1}$, a $\mathrm{BH}$ forms directly after an $\mathrm{SN}$, whereas in $\mathcal{R}_{\mathrm{MTBHB}, 2}$, an NS forms first and after a period of mass accretion reaches a critical mass $\left(M_{\max , \mathrm{NS}}\right)$ and collapses to a $\mathrm{BH}$. The critical mass is in general not well constrained and depends on the applied equation of state and rotation (e.g., Kalogera \& Baym 1996). In our simulations, a value of $M_{\text {max,NS }}=2.5 M_{\odot}$ was adopted. 


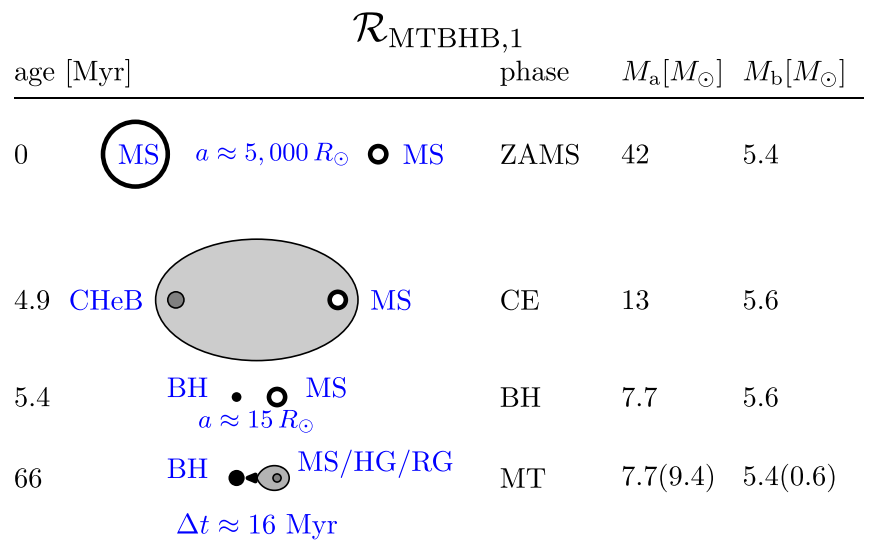

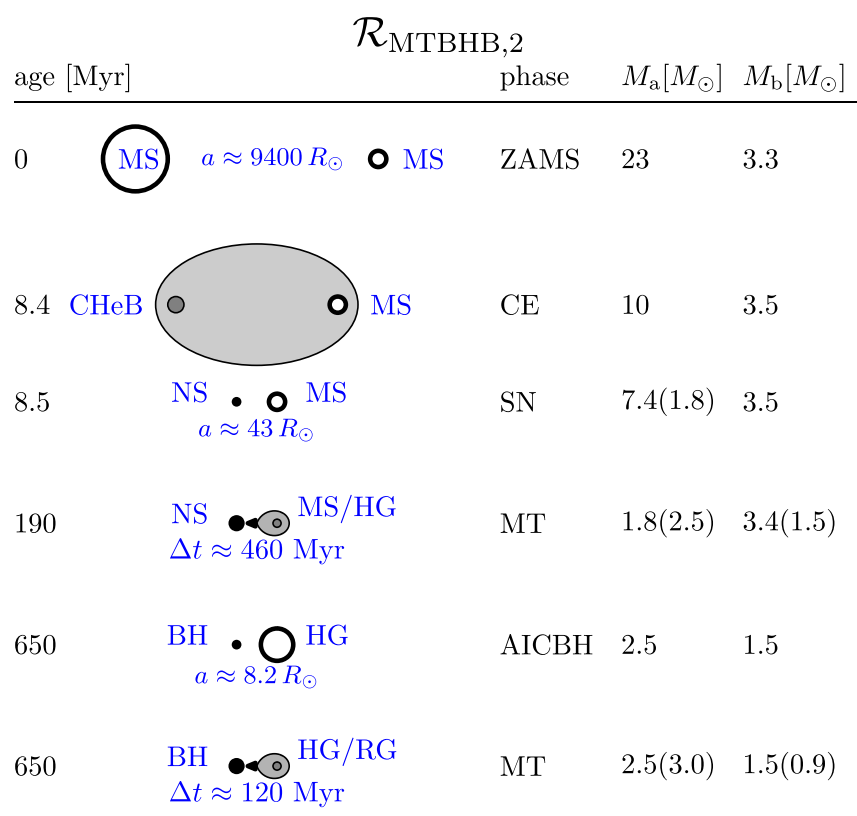

Figure 12. Evolution toward the formation of a typical MTBHB (routes $\mathcal{R}_{\mathrm{MTBHB}, 1}$ and $\mathcal{R}_{\mathrm{MTBHB}, 2}$ ). For descriptions of abbreviations and parameters, see Figure 2. Additionally: $\mathrm{CHeB}$, core helium burning; RG, red giant; $\mathrm{CE}$, common envelope; $\mathrm{AICBH}$, accretion-induced collapse of an NS into a $\mathrm{BH}$.

An example system that becomes an MTBHB through the $\mathcal{R}_{\text {MTBHB,1 }}$ route begins its evolution having ZAMS masses of $42 M_{\odot}$ and $5.4 M_{\odot}$ (Figure 12, upper plot). The separation is moderate, $a_{\text {ZAMS }} \approx 5000 R_{\odot}$. In $\sim 4.9 \mathrm{Myr}$, the primary evolves off the MS and commences a CE phase. Afterward, the separation is reduced to $\sim 13 R_{\odot}$. After an additional $0.5 \mathrm{Myr}$, the primary becomes a $\mathrm{BH}$ receiving a small $\mathrm{NK}$. The companion needs an additional $\sim 60 \mathrm{Myr}$ to expand through nuclear evolution and fill its Roche lobe. The MT is prolonged for $16 \mathrm{Myr}$, during which time the companion loses $~ 90 \%$ of its mass and evolves off the MS. The BH accretes about $1.5 M_{\odot}$.

As far as the $\mathcal{R}_{\text {MTBHB,2 }}$ route is concerned (Figure 12, lower plot), the initial masses on ZAMS are much smaller and in a typical case equal $\sim 23 M_{\odot}$ and $\sim 3.3 M_{\odot}$. The initial separation is large $\left(a \approx 9400 R_{\odot}\right)$. Similarly to $\mathcal{R}_{\mathrm{MTBHB}, 1}$, the primary evolves off the MS and commences a CE phase while being a $\sim 10 M_{\odot} \mathrm{CHeB}$ star. The outcome is a compact binary $\left(a \approx 40 R_{\odot}\right)$ composed of a $\sim 7.9 M_{\odot} \mathrm{HeS}$ and $\sim 3.5 M_{\odot} \mathrm{MS}$ star. The primary is not heavy enough to form a BH. Instead, in $100 \mathrm{kyr}$, it forms a heavy $\left(\sim 1.8 M_{\odot}\right)$ NS with a strong NK. The
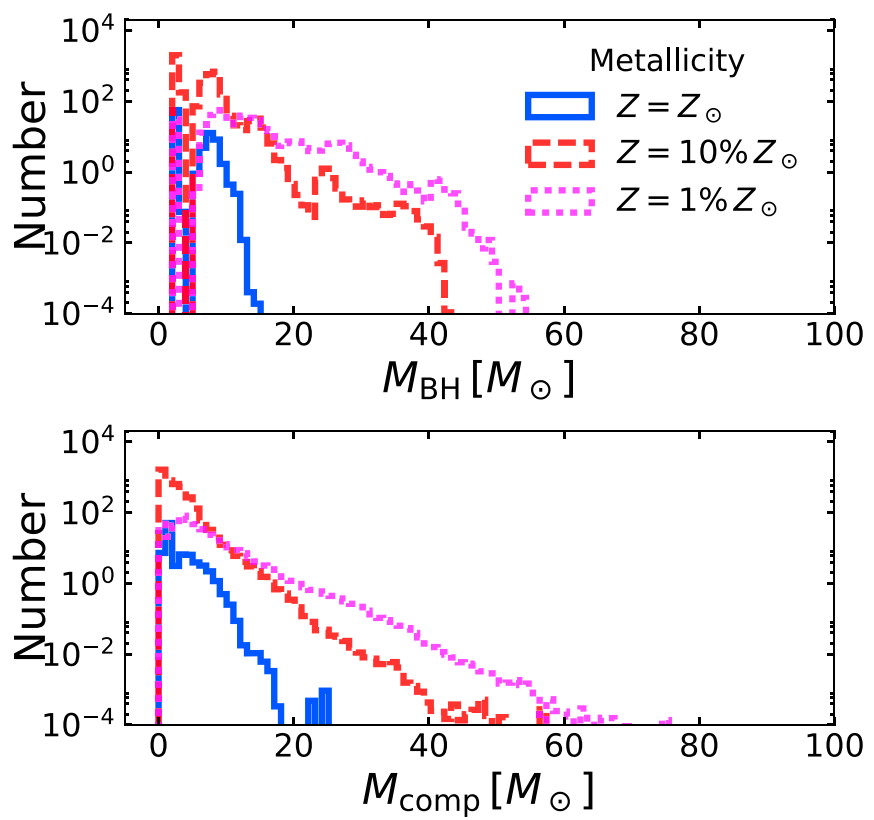

Figure 13. Distributions of BH masses (upper plot) and companion masses (lower plot) in mass-transferring (RLOF) binaries for three tested metallicities: $Z=Z_{\odot}\left(\right.$ STD model), $Z=10 \% Z_{\odot}($ mid- $Z$ model $)$, and $Z=1 \% Z_{\odot}($ low- $Z$ model).

orbit becomes highly elongated ( $a \approx 200 R_{\odot}, e \approx 0.96$ ). The secondary, which is now the more massive star, expands through nuclear evolution and after $\sim 200 \mathrm{Myr}$ fills its Roche lobe during periastron passage, and MT begins. In such a situation, we assume that the orbit is immediately circularized. In $\sim 500 \mathrm{Myr}$, the NS accretes $>0.7 M_{\odot}$, the critical mass is reached, and it collapses to a low-mass $\left(2.5 M_{\odot}\right) \mathrm{BH}$. Afterward, the MT restarts when the secondary is evolving off the MS. Finally, the $\mathrm{BH}$ grows to a mass of $\sim 3 M_{\odot}$.

Figure 13 shows distributions of masses for $\mathrm{BHs}$ and companions. The leftmost peak (for both $\mathrm{BH}$ and companion distributions) is composed of $\mathrm{BHs}$ formed through the $\mathcal{R}_{\text {MTBHB,2 }}$ route, whereas heavier $\mathrm{BHs}$ and companions represent the $\mathcal{R}_{\mathrm{MTBHB}, 1}$ route. The maximal $\mathrm{BH}$ masses naturally exceed those for wide BHBs or expected from single-star evolution (Belczynski et al. 2010a). The heaviest companions are usually accompanied by the heaviest BHs, which allow them to avoid extreme mass ratios, which in our calculations are assumed to lead to dynamic instability during MT.

Models mid- $Z$ and low- $Z$ predict larger populations of MTBHBs than the STD model. In lower metallicity environments, it is easier to produce heavier BHs, so the companion's Roche lobes are relatively smaller. Also, the nuclear expansion is slower, which allows for longer phases of stable MT.

Typical initial center-of-mass velocities $\left(v_{\mathrm{COM}, 0}\right)$ are smaller than $20 \mathrm{~km} \mathrm{~s}^{-1}$. This is a result of the fact that both heavy BHs $\left(M \gtrsim 40 M_{\odot}\right)$ and heavy NSs $\left(M>1.8 M_{\odot}\right)$ present in MTBHBs formed through channels $\mathcal{R}_{\mathrm{MTBHB}, 1}$ and $\mathcal{R}_{\mathrm{MTBHB}, 2}$, respectively, and have low or negligible NKs. The exceptions are models with higher average $\mathrm{NKs}\left(\mathrm{NK}_{\mathrm{R}}\right.$ and $\left.\mathrm{NK}_{\mathrm{BE}}\right)$, which give much higher $v_{\mathrm{COM}, 0}\left(50-100 \mathrm{~km} \mathrm{~s}^{-1}\right)$. We note that velocities around $100 \mathrm{~km} \mathrm{~s}^{-1}$ are also attainable in models with a standard NK distribution (e.g., STD) in systems with lighter BHs $\left(M_{\mathrm{BH}} \approx 6 M_{\odot}\right)$. 


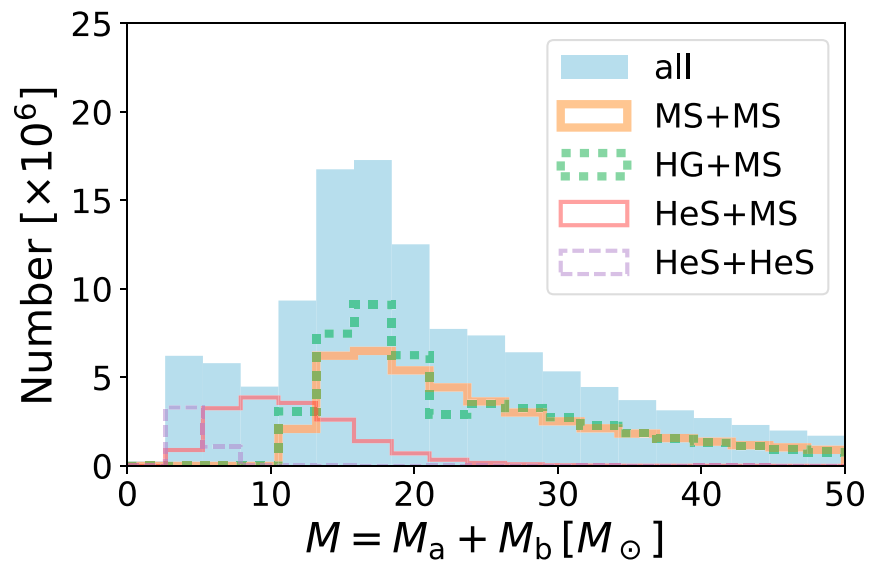

Figure 14. Distribution of the total binary masses prior to merger for the STD model. Only the main types of involved binaries are presented separately. The high-mass tail extends monotonically to $\sim 215 M_{\odot}$. Designations stand for MS, main sequence; HG, Hertzsprung gap; HeS, helium star.

\subsection{Mergers}

Mergers are a frequent outcome in population synthesis calculations of isolated binaries due to a failed CE ejection. Our results show that most typically (more than 95\% of cases) mergers occur when the components are on $\mathrm{MS}, \mathrm{HG}$, or are HeS (Figure 14). Although our results are not representative for the entire binary star population, as we include only binaries with an initial primary's mass in the range $10-150 M_{\odot}$, we include all potential progenitors of SBHs from merging binaries for which $M_{\mathrm{ZAMS}, \mathrm{a}}+M_{\mathrm{ZAMS}, \mathrm{b}} \geqslant 20 M_{\odot}$. We note that even if some lower-mass binaries may produce a $\mathrm{BH}$ predecessor after a merger, the poorly understood merger physics does not allow us to include such cases in the following analysis. Throughout the paper, we use a designation $\mathrm{mSBH}$ to distinguish SBHs formed from merger products from those originating from disrupted binaries.

In order to include merger products in the $\mathrm{BH}$ population, we have implemented a simplified formalism of Olejak et al. (2019; see Section 2.2). After merger, the products were evolved as single stars until a compact object is formed or reaching the age of $15 \mathrm{Gyr}$. This way, we were able to roughly estimate the population of $\mathrm{mSBHs}$. According to our simulations, the minimal ZAMS mass that produces a $\mathrm{BH}$ in singlestar evolution is between 19 and $22 M_{\odot}$ depending on the metallicity. Similarly, the minimal zero-age helium mainsequence mass that produces a $\mathrm{BH}$ is $\sim 9 M_{\odot}$ (e.g., Woosley 2019). In a typical case (MS+MS and $\mathrm{HG}+\mathrm{MS}$ mergers), there are no interactions prior to merger.

The predicted numbers of BHs for all tested models are provided in Table 2 . The model dependence is very low, with $\sim(3.6-6.6) \times 10^{7} \mathrm{BHs}$ originating from mergers, except flat IMF and steep IMF models, where the contrast is significant $\left(1.1 \times 10^{8}\right.$ and $1.6 \times 10^{7}$, respectively), due to a different (higher or lower, respectively) number of massive stars on ZAMS.

The mass distribution (Figure 15) shows a major peak at $\sim 7.5 M_{\odot}\left(\sim 15 M_{\odot}\right.$ for mid- $Z$ and low- $Z$ models $)$. The distribution noticeably differs from distributions of $\mathrm{BH}$ masses obtained through other formation channels. Particularly interesting is the presence of BHs inside the Mass Gap ( 2.5-5 $\left.M_{\odot}\right)$, where no compact objects have been detected through observations (e.g., Bailyn et al. 1998; Özel et al. 2010;

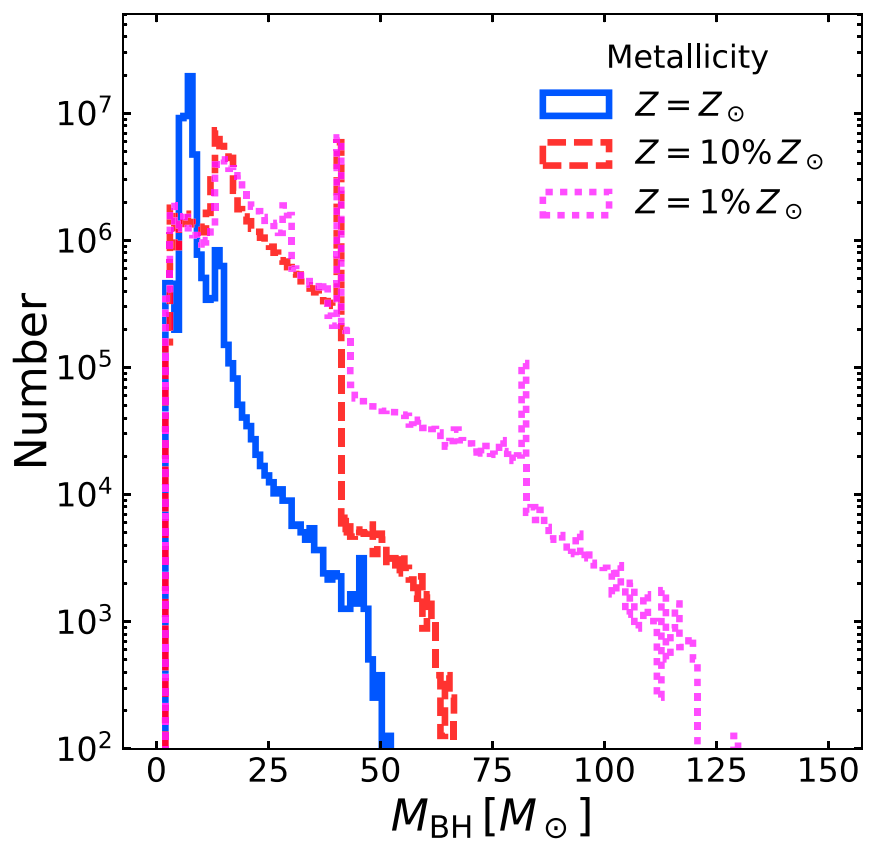

Figure 15. Distribution of $\mathrm{BH}$ masses from postmerger products according to the model by Olejak et al. (2019; see Section 2.2). A notable feature is the presence of BHs inside the Mass Gap $\left(2.5-5 M_{\odot}\right)$ and BHs much heavier than typical masses obtained through other evolutionary channels (e.g., $M_{\mathrm{BH}}>40 M_{\odot}$ for the STD model, or $M_{\mathrm{BH}}>70 M_{\odot} / 120 M_{\odot}$ for mid- $Z /$ low $-Z$ models).

Farr et al. 2011). These BHs mainly originate from mergers of NSs with HeSs, thus exceeding the maximal mass for an NS $\left(M_{\text {max,NS }}\right)$. In a typical case, the component masses on ZAMS are $\sim 10 M_{\odot}$ and $\sim 9.6 M_{\odot}$. The primary, being slightly heavier, evolves faster and in $\sim 24 \mathrm{Myr}$ fills its Roche lobe while expanding on the HG. The mass transfer leads to a mass reversal, with the primary becoming a $2.2 M_{\odot} \mathrm{HeS}$, whereas the secondary grows to $14 M_{\odot}$, still remaining on the MS. In about $4 \mathrm{Myr}$, the primary explodes as an electron capture supernova and forms an NS with a mass of $1.26 M_{\odot}$ and negligible NK. The separation is $770 R_{\odot}$ at that moment. The secondary needs an additional $1 \mathrm{Myr}$ to fill its Roche lobe during a $\mathrm{CHeB}$ phase. The mass ratio is extreme, with the donor being 10 times heavier then the accretor, which leads to a $\mathrm{CE}$ and, as a consequence, the merger of both stars. Finally, according to our prescription, a $2.9 M_{\odot} \mathrm{BH}$ forms. Such lowmass, free-floating $\mathrm{BHs}$ can be detectable by microlensing methods (e.g., Wyrzykowski et al. 2015). We note that the formation of low-mass BHs through this channel heavily depends on our assumptions concerning mergers (see Section 2.2) and, particularly, the fraction of the HeS that is actually accreted onto the NS.

Another striking feature of the distribution is the presence of high-mass tails exceeding up to $\sim 50 M_{\odot}, \sim 80 M_{\odot}$, or $\sim 130 M_{\odot}$ for STD, mid- $Z$, and low- $Z$ models, respectively (Figure 15). Typically, in the STD model, progenitors of such massive BHs are formed from a merger of a $\sim 25 M_{\odot}$ HeS with a $46 M_{\odot}$ MS star, which leaves a $\sim 43 M_{\odot}$ HeS as an outcome. Such a massive $\mathrm{HeS}$ collapses directly to a $\mathrm{BH}$ with $\sim 40 M_{\odot}$ mass. The evolution for massive $\mathrm{BHs}$ in other metallicities is analogous. These massive stars, although rare, may produce significantly stronger microlensing events than do those with typical masses $\left(\sim 7-8 M_{\odot}\right)$. We note that Spera et al. (2019) obtained even heavier SBHs as a result of stellar 
mergers using a different prescription for postmerger stellar parameters. In contrast to our results, their heaviest BHs (up to $\sim 145 M_{\odot}$ for metallicity $Z=0.0001$ ) originate from mergers of two MS stars.

BHs originating from mergers will typically have very low initial velocities $\left(v_{\mathrm{BH}, 0}\right)$. According to our results, more than $75 \%$ of the postmerger BHs will have no additional velocity over that resulting from the movement in the galactic potential $\left(v_{\mathrm{BH}, 0} \approx 0\right)$. Only BHs originating from double NS mergers may obtain high $v_{\mathrm{BH}, 0}$ (above $100 \mathrm{~km} \mathrm{~s}^{-1}$ ); however, they constitute only $<0.1 \%$ of all postmerger BHs. We note that asymmetries in the merger process and mass ejections may be a source of significant NKs, but they are not involved in this study.

\section{Application: Microlensing of Bulge Stars by Isolated BHs}

The gravitational microlensing effect is an amplification of the light of a distant object by another object (luminous or not) aligned in the line of sight toward the source (Paczynski 1996). Unfortunately, lens mass measurement, which is necessary to decide on its stellar or $\mathrm{BH}$ nature, is typically prone to parameter degeneracies (e.g., Wozniak et al. 2001; Sumi et al. 2013; Wyrzykowski et al. 2015; Mróz et al. 2017), and BH lenses can be found only in particular circumstances (e.g., Bennett et al. 2002; Dong et al. 2007; Wyrzykowski et al. 2016; Rybicki et al. 2018). On the other hand, population studies allow us to estimate the expected number of microlensing events with $\mathrm{BH}$ lenses, which we do in the following analysis. Other similar attempts include studies by Osłowski et al. (2008) and Lu et al. (2019).

Due to the high star number density and relative proximity, the Galactic Bulge is a frequent direction of observations aimed at detecting microlensing events (e.g., Gould 2000; Wyrzykowski et al. 2015); therefore, here we also concentrate on this particular direction.

We make a series of simplifying assumptions. First of all, we assume that the source is always located in the bulge, which is represented by a flat disk of size $\theta_{\text {bulge }}=31$ square degrees, possessing $N_{\star, \text { bulge }}=1.5 \times 10^{8}$ stars. The tangential velocity distribution (radial velocity can be ignored in microlensing) is on average zero and has a dispersion $\sigma_{\text {bulge }, z}=\sigma_{\text {bulge }, y}=80 \mathrm{~km} \mathrm{~s}^{-1}$ in both directions, parallel ( $y$ axis) and perpendicular ( $z$ axis) to the galactic plane (Skowron et al. 2011).

Second, we assume that a $\mathrm{BH}$ lens is always located in the galactic disk, which has a total mass of $5 \times 10^{10} M_{\odot}$ (Licquia \& Newman 2015), and its stellar number density (we assume that BHs have the same distribution as stars in general) is described by

$$
\rho_{\text {star }} \propto e^{\frac{|z|}{0.3 \mathrm{kpc}}-\frac{R}{2.6 \mathrm{kpc}}}
$$

where $z$ is the distance from the galactic plane, and $R$ is the distance from the galactic center in the galactic plane, following Batista et al. (2011) and Skowron et al. (2011). We assume that components of the tangential velocity of stars in the disk are on average $v_{\text {disk,mean, } z}=0$ in the direction perpendicular to the galactic plane and $v_{\text {disk,mean, } y}=200 \mathrm{~km} \mathrm{~s}^{-1}$ in the galactic plane. The tangential velocity dispersion is assumed to be $\sigma_{\text {disk }, z}=$ $40 \mathrm{~km} \mathrm{~s}^{-1}$ perpendicular to the galactic plane and $\sigma_{\text {disk, } y}=$ $55 \mathrm{~km} \mathrm{~s}^{-1}$ parallel to the galactic plane.

In contrast to other studies, here we use the BHs from disrupted binaries and stellar mergers only, as BHs in binaries represent a small fraction $(<20 \%)$ of the total population (Table 2). Additionally, microlensing by a binary lens is more complex (caustic crossing, high amplification, and so on) and will be investigated in a separate study. Also, due to the assumed $100 \%$ binary fraction for stars heavier than $10 M_{\odot}$ on ZAMS, we do not expect any BHs formed through single-star evolution.

Stellar mergers may potentially constitute a significant part of the $\mathrm{BH}$ population (see Section 3.3), so it is necessary to include them in the calculation of the microlensing rate. In such a case, not only the number of BHs is important, but also their masses and proper motions. We calculate the final $\mathrm{BH}$ mass following the formulas of Olejak et al. (2019; see Section 2.2 for the postmerger mass and evolutionary phase). As far as the proper motions are concerned, we assume that the $v_{\mathrm{BH}, 0}$ of the postmerger star is equal to the premerger center-of-mass velocity of the binary; that is, merger occurs without a kick.

We use the Monte Carlo method to sample the spatial and velocity distributions of both lenses and sources. In our analysis, we include only lenses that were localized between the observer, assumed to be located at $x_{\odot}=8.3 \mathrm{kpc}$ from the Galactic center, and the Galactic Bulge, ignoring less likely lenses and sources from the far Galactic disk.

An angular Einstein radius of a lens depends on its mass $\left(M_{\mathrm{L}}\right)$ and distances between the observer and the lens $\left(D_{\mathrm{L}}\right)$ or the source $\left(D_{\mathrm{S}}\right)$ :

$$
\theta_{\mathrm{E}}=\sqrt{\kappa M_{\mathrm{L}} \pi_{\text {rel }}}, \pi_{\text {rel }}=\frac{1}{D_{\mathrm{L}}}-\frac{1}{D_{\mathrm{S}}},
$$

where $\kappa=\frac{4 G}{c^{2}} \approx 8.1 \frac{\mathrm{mas}}{M_{\odot}}$. If the relative proper motion between the lens and the source is $\mu_{\text {rel }}=\mu_{\mathrm{L}}-\boldsymbol{\mu}_{\mathrm{S}}$, the source crosses the Einstein radius in a time called the Einstein radius crossing time, or simply the event's timescale, $t_{\mathrm{E}}=\frac{\theta_{\mathrm{E}}}{\left|\mu_{\text {rel }}\right|}$.

The relative proper motion is computed using the velocities of the lens and the source as

$$
\mu_{\mathrm{rel}, y / z}=\frac{v_{\mathrm{rel}, y / z}}{x_{\odot}-x}
$$

and

$$
\begin{aligned}
v_{\text {rel }, y / z}= & v_{\mathrm{BH}, y / z}-v_{\text {Earth }, y / z} \\
& -\left(v_{\text {source }, y / z}-v_{\text {Earth }, y / z}\right)\left(1-\frac{x}{x_{\odot}}\right),
\end{aligned}
$$

where $v_{\mathrm{rel}, y / z}$ is a component of the relative velocity along the $y / z$ axis. Here, $v_{\mathrm{BH}, y / z}, v_{\text {source }, y / z}$, and $v_{\text {Earth }, y / z}$ are the velocities of the $\mathrm{BH}$, the source, and the observer, along the $y / z$ axis, respectively. The observer's velocity was assumed to be $v_{\text {Earth }, ~} y / z=230 / 15.5 \mathrm{~km} \mathrm{~s}^{-1}$. Also, $x=x_{\odot}-D_{\mathrm{L}}$ is the distance of the lens from the Galactic center.

Finally, we calculated an estimated number of microlensing events $\left(E\left(N_{\mathrm{ML}}\right)\right)$ during $t=1 \mathrm{yr}$ as (assuming $\theta_{\mathrm{E}}$ is small)

$$
\mathrm{E}\left(N_{\mathrm{ML}}\right)=\frac{\sum_{i}\left(2 \theta_{\mathrm{E}, \mathrm{i}} \frac{v_{\mathrm{rel}, \mathrm{i}}}{x_{\odot}-x_{\mathrm{i}}} t+\pi \theta_{\mathrm{E}, \mathrm{i}}^{2}\right)}{\Omega_{\text {bulge }}} N_{\star, \text { bulge }},
$$

where $\theta_{\mathrm{E}, \mathrm{i}}$ is the Einstein radius generated by the $i$ th $\mathrm{BH}$, $v_{\text {rel, } \mathrm{i}}=\sqrt{v_{\mathrm{rel}, \mathrm{x}, \mathrm{i}}^{2}+v_{\mathrm{rel}, \mathrm{y}, \mathrm{i}}^{2}}$ is the relative tangential velocity of the $i$ th $\mathrm{BH}$, and $\Omega_{\text {bulge }}$ is the considered area of the bulge in 


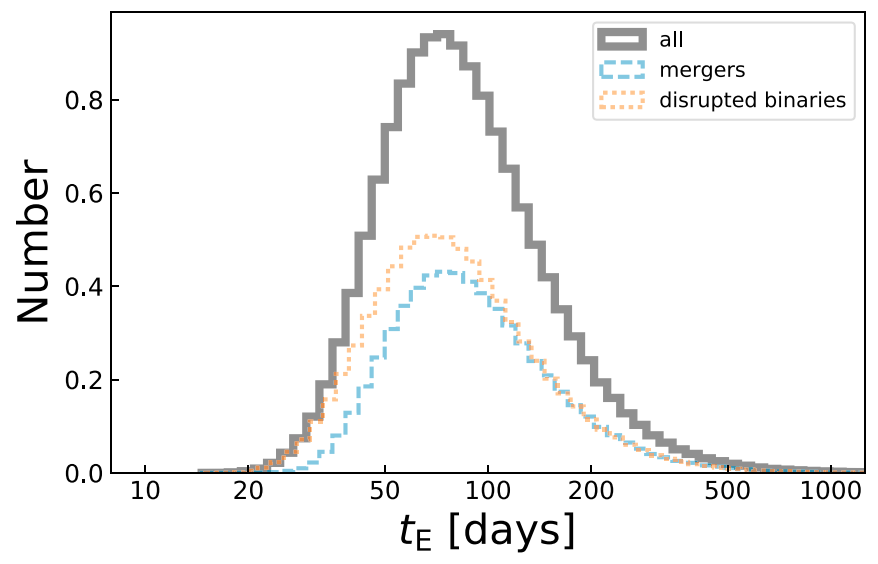

Figure 16. Distribution of microlensing timescales (Einstein ring crossing times, $\left.t_{\mathrm{E}}=r_{\mathrm{E}} / v_{\text {rel }}\right)$ for $\mathrm{BH}$ lenses from binaries. We make a division between $\mathrm{BHs}$ originating from stellar mergers and those from disrupted binaries. The average values of $t_{\mathrm{E}}$ for BHs from mergers ( $\sim 108$ days) are relatively higher than for BHs from disrupted binaries ( $\sim 97$ days).

steradians. The summation goes over all of the included BHs, that is, localized between the observer and the bulge.

If we assume that the stellar mass of the Galactic disk is $\sim 5 \times 10^{10} M_{\odot}$ (Licquia \& Newman 2015) and we take the number of stars in the bulge as $N_{\star \text {, bulge }}=1.5 \times 10^{8}$ from the number of monitored stars by the OGLE-III survey covering 31 sq. deg. of the bulge (Wyrzykowski et al. 2015), we predict that there might be as many as $\sim 14 \mathrm{yr}^{-1}$ microlensing events due to BHs. In the so-far analyzed OGLE-IV data (Udalski et al. 2015; Mroz et al. 2019), which covers 160 sq. deg. and monitors 400 million sources, the number of expected events due to $\mathrm{BHs}$ grows to $\sim 26 \mathrm{yr}^{-1}$. Here we include only events with characteristic time $t_{\mathrm{E}}$ in the range 1-1000 days, which includes most of the events $(>95 \%)$. Although $\mathrm{BHs}$ from disrupted binaries have higher velocities and, therefore, a higher chance for microlensing, BHs from disrupted binaries (dSBH; Section 3.1) dominate the rate ( $\sim 55 \%$ of events), due to higher masses and greater abundance.

For a given source and lens distances and their velocities, we computed $\theta_{\mathrm{E}}$ and $\mu_{\text {rel }}$ and hence the distribution of timescales ( $t_{\mathrm{E}}$, Figure 16). The typical values of $t_{\mathrm{E}}$ are on average $\sim 97$ days for BHs originating from disrupted binaries $(\mathrm{dSBH}$; Section 3.1) and $\sim 108$ days for those originating from stellar mergers (mSBH; Section 3.3).

Events with such timescales are detectable in current microlensing surveys with an efficiency of more than $75 \%$ (e.g., Sumi et al. 2013; Wyrzykowski et al. 2015). This means in the OGLE-III data covering $8 \mathrm{yr}$ of monitoring of 150 million stars there should be about 84 events due to BHs. Because, as shown above, these tend to have longer timescales, the annual Earth motion (the parallax effect) is likely to cause distortion of the standard microlensing light curve; therefore, it is possible that some fraction of those 84 events are not in the Wyrzykowski et al. (2015) sample of standard events. Indeed, Wyrzykowski et al. (2016) have identified 59 OGLE-III events with significant parallax effect, among which 13 are likely due to NSs and BHs. The improved analysis of Wyrzykowski \& Mandel (2019) has increased the number of NS and BH lenses to 18 in that sample. The remaining undetected $\mathrm{BH}$ lenses might still await discovery in the OGLE-III data with moderate and small annual parallax signatures.

\section{Discussion \\ 5.1. Detectability of Single BHs}

Although the predicted number of SBHs in MWEG (both $\mathrm{dSBH}$ and $\mathrm{mSBH})$ is very significant $\left(3.2 \times 10^{7}\right.$ to $\left.2.5 \times 10^{8}\right)$, these objects still evade detection. Shapiro \& Teukolsky (1983) have already estimated that BHs in the Galaxy should be counted in millions. Timmes et al. (1996) estimated that there are even $1.4 \times 10^{9} \mathrm{BHs}$ in the Galaxy. Samland (1998) calculated the $\mathrm{BH}$ population to be $1.8 \times 10^{8}$. They included the changes in SFR, but excluded binary stars from their analysis. It was proposed that SBHs may be detected when interacting with interstellar matter (Shvartsman 1971; Agol \& Kamionkowski 2002; Barkov et al. 2012; Tsuna et al. 2018; Tsuna \& Kawanaka 2019); however, the estimated X-ray emission from an accreting SBH is very small $\left(<10^{35} \mathrm{erg} \mathrm{s}^{-1}\right.$; e.g., Barkov et al. 2012). Such faint sources may be potentially detected only in our Galaxy. Matsumoto et al. (2018) suggested that the accretion onto an SBH from the interstellar medium may not be necessarily spherical and result in the formation of an accretion disk. In such disks, the same instabilities may arise as in X-ray novae, resulting in a transient behavior with outburst luminosities reaching $\sim 10^{38} \mathrm{erg} \mathrm{s}^{-1}$.

SBHs can be also detected as gravitational microlenses (Agol et al. 2002; Wyrzykowski et al. 2016; Rybicki et al. 2018). As shown in this paper, SBHs may have very high velocities that increase the chance for a microlensing event. On the other hand, a significant part of DCOs $\left(\mathcal{R}_{\mathrm{DCO}, 2}\right.$ channel) have separations small enough to act as a single, very massive lens, with the mass as the sum of the masses of individual components. In combination with small proper motions of such binaries, they may generate long-lasting $\left(t_{\mathrm{E}} \sim 1 \mathrm{yr}\right)$ events; however, we note that undisrupted compact DCOs make up less than $15 \%$ of all BHs in the Milky Way.

Although no SBHs were detected so far, a few observational methods were proposed. The predicted number of observations in future X-ray missions is strongly dependent on the $\mathrm{BH}$ velocities and accretion models. Future missions may help to improve the constraints on this physics. Nevertheless, detectability of SBHs in X-rays is strongly spoiled by background active galactic nuclei, hard coronal emitters, and cataclysmic variables (Motch \& Pakull 2012). Another possibility, to which more attention is paid in this work, is the detection of SBHs with microlensing (Paczynski 1986). The first candidates were proposed in Bennett et al. (2002) and Mao et al. (2002) and were followed up with X-ray observations, but no signal from ISM accretion was detected (Maeda et al. 2005; Nucita et al. 2006). Recently, several candidates were proposed (Wyrzykowski et al. 2016), but the lens's mass estimates are heavily degenerated by the lack of measurement of relative velocities. Consequently, WDs or NSs cannot be fully ruled out. For the currently ongoing events, there is an opportunity for degeneracy breaking thanks to astrometric measurements from optical interferometry (Dong et al. 2019) or Gaia (Rybicki et al. 2018); however, this will only be possible for the brightest events. In the near future with the LSST, it should be possible to observe thousands of microlensing events due to BHs and, therefore, to compare the stellar evolution predictions with the observed parameters of Galactic BHs. 


\subsection{Detectability of $\mathrm{BHs}$ in Binaries}

All dynamically confirmed galactic BHs were detected in XRBs. We note that a $\mathrm{BH}$ candidate was also detected in a noninteracting binary (Thompson et al. 2018), and many more are expected to be seen by Gaia (Breivik et al. 2017; Mashian \& Loeb 2017; Yamaguchi et al. 2018). In XRBs, a compact object accretes mass from its companion, which results in formation of an accretion disk and production of highly energetic radiation. If the companion is observable, radial velocities can be measured and the mass function can be estimated. Unfortunately, the majority of XRBs containing BHs are transient systems and thus are visible only during outbursts, and only a few recurring systems were observed. What is more, typical transient systems are characterized by low-mass donors $\left(M_{\text {don }} \lesssim 2 M_{\odot}\right)$ and therefore are undetectable from extragalactic distances and outshined by the disk during an outburst. In $19 \mathrm{XRBs}$, BHs were dynamically confirmed (e.g., Wiktorowicz et al. 2014); however, there are many more candidates (Corral-Santana et al. 2016).

Gravitational wave emission from double $\mathrm{BH}$ mergers may be perceived as a detection of BHs. Up to now, 10 binary $\mathrm{BH}$ merger events were detected by LIGO/Virgo (The LIGO Scientific Collaboration et al. 2019). Using a different pipeline, Venumadhav et al. (2019) found six additional sources in publicly available data from the second observing run. Notably, in spite of one double NS merger detection (Abbott et al. 2017b), no BH+NS mergers were detected. The masses of premerger $\mathrm{BHs}$ range from $\sim 7.7 M_{\odot}$ to $\sim 50.6 M_{\odot}$ with the total binary mass as high as $\sim 85.1 M_{\odot}$ (The LIGO Scientific Collaboration et al. 2019). Many tens of detections are expected from the next observational runs (The LIGO Scientific Collaboration et al. 2019), and several candidates have already been found (e.g., Singer 2019).

BHs in binaries may be detected also through microlensing. They may lens differently depending on the compactness of the binary. If the separation is significantly smaller than the total mass Einstein radius, the system will act as a single lens with a mass equal to the sum of the components' masses. On the other hand, if the system is very wide (many Einstein radii), a $\mathrm{BH}$ will act as a single lens unaffected by a remote companion. Between these two options, we have systems that act as a binary lens with all of the effects connected with caustics crossing. Up to now, no microlensing events with $\mathrm{BH}$ lenses were confirmed. Although there are several candidates (e.g., Miyake et al. 2012; Shvartzvald et al. 2015; Wyrzykowski et al. 2016), parameter degeneracies make it impossible to infer mass and thus distinguish between a $\mathrm{BH}$ and other lowluminosity objects like WDs.

\subsection{Comparison with Previous Estimates of Microlensing Rates by $\mathrm{BHs}$}

Our prediction of $\sim 14$ microlensing events due to $\mathrm{BHs}$ in a year stays in contrast to previous estimates. Gould (2000) analytically calculated that only $1 \%$ of microlensing events in the direction of the bulge are due to $\mathrm{BH}$ lenses, which gives about 30 events in OGLE-III (three to four per year). There are several reasons for this contradiction. First of all, Gould (2000) concentrates on BH lenses in the bulge, whereas our lenses are in the disk. The $\pi_{\text {rel }}$ (see Equation (5)) is typically smaller for bulge lenses, and thus also the Einstein radius, which is proportional to the probability of microlensing. Second,
Gould (2000) assumed $40 M_{\odot}$ as a minimal ZAMS mass producing a $\mathrm{BH}$ remnant, whereas in our calculations we take $\sim 22 M_{\odot}$ (for solar metallicity). Consequently, they obtained many fewer BHs from the same initial population. Moreover, BHs in Gould (2000) follow the velocity distribution of stars, whereas in our simulations BHs obtain additionally a kick (including NK, Blaauw kick, and the kick from binary disruption). Third, the binary systems were ignored in the work of Gould (2000), while here we show that the binary systems could potentially contribute significantly to the population of lensing BHs.

In a more recent work, Rybicki et al. (2018) used the same population synthesis StarTrack code to estimate the microlensing rate events due to BHs. However, they focus on astrometric microlensing events, that is, events in which not only photometric observation is possible, but also astrometric signals can be detected with the Gaia space mission. They estimated there should be a few such events per year. About an order of magnitude of difference between our results stems from the fact that only $\sim 5 \%$ of bulge stars $\left(5 \times 10^{6}\right.$ in their work) are bright enough to potentially generate a detectable astrometric microlensing event. In contrast, we included all bulge stars as potential sources $\left(1.5 \times 10^{8}\right)$, thus 30 times more, which results in a significantly higher estimated rate. Moreover, Rybicki et al. (2018) used averaged or typical values to calculate the rates, whereas in this paper we populate the Galaxy with stars, evolve them to the formation of a $\mathrm{BH}$, and calculate the rates directly.

We note that in our approach to estimating the microlensing rates we made a number of simplifications. Especially, we used only one model from eight analyzed in this paper. Additionally, we chose a simple model of BH distribution in the Galaxy. For example, the model of Robin et al. (2003), which was used in Rybicki et al. (2018), gives a microlensing rate by BHs of $\sim 9 \mathrm{yr}^{-1}$, so nearly two times smaller than the rate obtained for the distribution described by Equation (4). We also note that the rate may change by a factor of $\sim 5$ depending on the chosen evolutionary parameters, such as IMF, NKs, and metallicity.

\subsection{Hertzsprung Gap Donors in Common Envelope Evolution}

It is still not known if binaries undergoing $\mathrm{CE}$ events with $\mathrm{HG}$ donors will merge, due to the lack of a clear core-envelope boundary, or if they can survive, because the boundary develops during the $\mathrm{HG}$ phase. In order to check the importance of our assumption (model B-CE with $\mathrm{HG}$ donor always leads to a merger), we have also tested an opposite case (model A) where binaries are allowed to survive the CE phase even if the donor is on its HG. The results are presented in Table 7.

The difference in the total number of BHs is small $(\lesssim 10 \%)$, as most of the BHs form without a CE phase during the binary evolution. More pronounced is the higher number of DCOs (particularly the close ones), which may exceed two orders of magnitude in comparison to model $\mathrm{B}$ for higher NK models $\left(\mathrm{NK}_{\mathrm{R}}\right.$ and $\left.\mathrm{NK}_{\mathrm{BE}}\right)$. Some close $\mathrm{DCO}$ progenitors evolve through a $\mathrm{CE}$ phase, and in model A donors are frequently $\mathrm{HG}$ stars.

The number of mergers leading to the formation of a $\mathrm{BH}$ is also affected. In model $\mathrm{B}, \mathrm{CE}$ events with $\mathrm{HG}$ donors are treated as mergers, which is not always the case in model A. In consequence, there are significantly fewer (up to $33 \%$ ) $\mathrm{BHs}$ originating from stellar mergers in model $\mathrm{A}$ than in model $\mathrm{B}$. 
Table 7

Number of BHs in the Milky Way Equivalent Galaxy (Model A)

\begin{tabular}{|c|c|c|c|c|c|c|c|c|c|}
\hline \multirow{2}{*}{$\frac{\text { Model }}{\text { STD }}$} & \multirow{2}{*}{$\frac{N_{\mathrm{BH}, \mathrm{tot}}}{1.0 \times 10^{8}}$} & \multicolumn{2}{|c|}{$N_{\mathrm{dSBH}}$} & \multicolumn{2}{|c|}{$N_{\mathrm{BHB}}$} & \multicolumn{2}{|c|}{$N_{\mathrm{BH}, \mathrm{DCO}}$} & \multicolumn{2}{|c|}{$N_{\mathrm{mSBH}}$} \\
\hline & & $5.4 \times 10^{7}$ & $(54.2 \%)$ & $1.5 \times 10^{6}$ & $(1.5 \%)$ & $6.1 \times 10^{6}$ & $(6.1 \%)$ & $3.8 \times 10^{7}$ & $(38.2 \%)$ \\
\hline mid- $Z$ & $1.2 \times 10^{8}$ & $3.5 \times 10^{7}$ & $(29.9 \%)$ & $2.0 \times 10^{6}$ & $(1.7 \%)$ & $2.2 \times 10^{7}$ & $(18.8 \%)$ & $5.8 \times 10^{7}$ & $(49.6 \%)$ \\
\hline low-Z & $1.2 \times 10^{8}$ & $3.7 \times 10^{7}$ & $(30.7 \%)$ & $1.5 \times 10^{6}$ & $(1.2 \%)$ & $2.1 \times 10^{7}$ & $(17.4 \%)$ & $6.1 \times 10^{7}$ & $(50.6 \%)$ \\
\hline SSO & $1.0 \times 10^{8}$ & $6.6 \times 10^{7}$ & $(65.0 \%)$ & $3.4 \times 10^{6}$ & $(3.3 \%)$ & $8.1 \times 10^{6}$ & $(8.0 \%)$ & $2.4 \times 10^{7}$ & $(23.6 \%)$ \\
\hline $\mathrm{NK}_{\mathrm{R}}$ & $1.0 \times 10^{8}$ & $6.4 \times 10^{7}$ & $(63.8 \%)$ & $6.0 \times 10^{4}$ & $(0.1 \%)$ & $2.1 \times 10^{5}$ & $(0.2 \%)$ & $3.6 \times 10^{7}$ & $(35.9 \%)$ \\
\hline $\mathrm{NK}_{\mathrm{BE}}$ & $1.0 \times 10^{8}$ & $6.5 \times 10^{7}$ & $(64.3 \%)$ & $5.5 \times 10^{4}$ & $(0.1 \%)$ & $8.7 \times 10^{4}$ & $(0.1 \%)$ & $3.6 \times 10^{7}$ & $(35.6 \%)$ \\
\hline flat IMF & $2.5 \times 10^{8}$ & $1.4 \times 10^{8}$ & $(56.0 \%)$ & $3.2 \times 10^{6}$ & $(1.3 \%)$ & $1.6 \times 10^{7}$ & $(6.4 \%)$ & $9.1 \times 10^{7}$ & $(36.4 \%)$ \\
\hline steep IMF & $3.1 \times 10^{7}$ & $1.6 \times 10^{7}$ & $(50.9 \%)$ & $5.6 \times 10^{5}$ & $(1.8 \%)$ & $1.9 \times 10^{6}$ & $(6.0 \%)$ & $1.3 \times 10^{7}$ & $(41.3 \%)$ \\
\hline
\end{tabular}

Note. The same as Table 2, but for a model in which HG donors were allowed to survive the CE phase (see Section 5.4 for details).

As the total number of BHs is affected only slightly by the treatment of $\mathrm{HG}$ donors in $\mathrm{CE}$ events, we predict a negligible impact on the microlensing observations. However, it may be significant if events caused by close systems are considered.

\subsection{Binary Fraction}

In this work, we assumed that all stars heavier than $10 M_{\odot}$ are born in binaries. Such a high binary fraction (100\%) for massive stars is supported by observations, which suggest their binary fraction to be higher than $90 \%$ (Sana et al. 2012). For lower-mass stars, we assumed an equal number of binaries and single stars on ZAMS (the binary fraction equals $50 \%$ ).

Nonetheless, we agree that the binary fraction may not change in such a drastic way when the mass of the primary increases. More probably, the binary fraction increases continuously. Therefore, a fraction of massive stars that are heavy enough to produce BHs $\left(M_{\text {ZAMS }} \gtrsim 20 M_{\odot}\right)$ may actually form without companions. If included in our simulations, these stars could increase the relative fraction of SBHs in the entire $\mathrm{BH}$ population. However, the total number of $\mathrm{BHs}$ is expected to be lower because the flat mass ratio distribution in binaries gives a higher average mass of two stars in a binary, and thus more $\mathrm{BH}$ progenitors, than two single stars following the IMF relation. We note that mergers of binary systems of two massive stars (both heavy enough to form a $\mathrm{BH}$ in a single star evolution) may decrease the total number of BHs, but mergers of NS progenitors $\left(M_{\text {zams }} \approx 8-20 M_{\odot}\right)$ may become heavy enough to form a $\mathrm{BH}$ in posterior evolution, thus increasing the total number of BHs.

Many of the massive binaries may be part of triples and higher-order systems (e.g., Toonen et al. 2016). Even 50\% of massive (OB type) stars may exist in triples (e.g., Sana et al. 2014), but the inner binary in a hierarchical triple system may evolve effectively isolated from the third star, so estimating the significance of higher-order systems on $\mathrm{BH}$ populations is complicated. Of particular interest are mergers induced by the Kozai-Lidov mechanism in stellar triples (e.g., Antonini et al. 2017). However, higher-order systems are not understood yet well enough to be included in population synthesis modeling.

\section{Summary}

In this paper, we analyzed the general properties of synthetic $\mathrm{BH}$ populations in different stellar environments represented by models that differ in the metallicity, initial parameter distributions, and NK prescriptions. We note that the results are applicable for further studies like predictions for present and future survey missions or in-depth analysis of specific $\mathrm{BH}$ populations (e.g., the Milky Way galaxy). We particularly focused on BHs originating from disrupted binaries and stellar mergers, which were frequently omitted in previous studies. We find that those $\mathrm{BHs}$ constitute a majority of the total $\mathrm{BH}$ population.

Particularly, we show that SBHs dominate the total population of $\mathrm{BHs}$ ( $\gtrsim 83 \%$ of all $\mathrm{BHs}$ ), even though massive stars form predominantly in binaries. Both binary disruptions and stellar mergers are important, and the predicted number of BHs is only slightly affected (up to a factor of $\sim 3$ ) by a chosen model. Although BHs in binaries constitute only a small part $(\lesssim 17 \%)$ of the population, their number is heavily dependent (about two orders of magnitude) on the adopted model parameters (especially $\mathrm{NK}$ prescription and $\mathrm{SFH}$ ), so new observations may significantly help to constrain these parameters and better understand evolutionary processes (e.g., Maccarone et al. 2019).

Using our results, we calculated the expected rate of microlensing events with $\mathrm{BH}$ lenses in the direction of the Galactic Bulge. We expect as many as $\sim 14$ such events per year with average crossing times of around 100 days in the OGLE-III footprint and about 26 in the OGLE-IV data. Only some of these events may be observable from Earth, due to technical limitations (e.g., low luminosity, extinction). In this estimate, we have neglected BHs that remained in binaries, because the low fraction ( $\lesssim 17 \%$ of all BHs) and slow velocities (typically, $\lesssim 20 \mathrm{~km} \mathrm{~s}^{-1}$ ) allow us to suppose that their influence is small.

A grid of 54 models (including eight main models analyzed in this paper) is available in a free-access database. ${ }^{7}$ In our future work, we plan to utilize these data files in order to study microlensing by SBHs in the Galaxy and provide detailed predictions for surveys like Gaia or the Einstein Telescope.

We are thankful to the anonymous referee who helped to improve the paper and thousands of volunteers, who took part in the Universe@Home project ${ }^{8}$ and provided their computers for this research. We acknowledge the Polish NCN grant HARMONIA $2015 / 18 / \mathrm{M} / \mathrm{ST} 9 / 00544$ to $\mathrm{ŁW}$, OPTICON H2020 EC grant No. 730890, MNiSW grant DIR/WK/ 2018/12, and SONATA BIS 2 DEC-2012/07/E/ST9/ 01360. G.W. is partly supported by the Presidents International Fellowship Initiative (PIFI) of the Chinese Academy of Sciences under grant No. 2018PM0017 and by the Strategic Priority Research Program of the Chinese Academy of Science Multi-waveband Gravitational Wave Universe (grant

\footnotetext{
7 https://universeathome.pl/universe/bhdb.php

8 https://universeathome.pl/
} 
No. XDB23040000). K.B. and G.W. acknowledge NCN grant MAESTRO 2018/30/A/ST9/00050. M.C. and J.K. acknowledge support from the Netherlands Organisation for Scientific Research (NWO). We acknowledge the Polish NCN grant PRELUDIUM 2017/25/N/ST9/01253 to K.R. This work is partly supported by the National Key Program for Science and Technology Research and Development (grant No. 2016YFA0400704), and the National Natural Science Foundation of China (grant Nos. 11690024 and 11873056).

\section{ORCID iDs}

Łukasz Wyrzykowski (i) https://orcid.org/0000-00029658-6151

\section{References}

Abbott, B. P., Abbott, R., Abbott, T. D., et al. 2016a, ApJL, 818, L22 Abbott, B. P., Abbott, R., Abbott, T. D., et al. 2016b, PhRvL, 116, 241103 Abbott, B. P., Abbott, R., Abbott, T. D., et al. 2017a, PhRvL, 118, 221101 Abbott, B. P., Abbott, R., Abbott, T. D., et al. 2017b, PhRvL, 119, 141101 Abt, H. A. 1983, ARA\&A, 21, 343

Adams, S. M., Kochanek, C. S., Gerke, J. R., Stanek, K. Z., \& Dai, X. 2017, MNRAS, 468, 4968

Agol, E., \& Kamionkowski, M. 2002, MNRAS, 334, 553

Agol, E., Kamionkowski, M., Koopmans, L. V. E., \& Blandford, R. D. 2002, ApJL, 576, L131

Antonini, F., Toonen, S., \& Hamers, A. S. 2017, ApJ, 841, 77

Bailyn, C. D., Jain, R. K., Coppi, P., \& Orosz, J. A. 1998, ApJ, 499, 367

Bambi, C. 2018, AnP, 530, 1700430

Banerjee, S. 2017, MNRAS, 467, 524

Barkov, M. V., Khangulyan, D. V., \& Popov, S. B. 2012, MNRAS, 427, 589

Batista, V., Gould, A., Dieters, S., et al. 2011, A\&A, 529, A102

Belczynski, K., Bulik, T., Fryer, C. L., et al. 2010a, ApJ, 714, 1217

Belczynski, K., Bulik, T., \& Kluźniak, W. 2002a, ApJL, 567, L63

Belczynski, K., Dominik, M., Bulik, T., et al. 2010b, ApJL, 715, L138

Belczynski, K., Heger, A., Gladysz, W., et al. 2016a, A\&A, 594, A97

Belczynski, K., Holz, D. E., Bulik, T., \& O’Shaughnessy, R. 2016b, Natur, 534,512

Belczynski, K., Kalogera, V., \& Bulik, T. 2002b, ApJ, 572, 407

Belczynski, K., Kalogera, V., Rasio, F. A., et al. 2008, ApJS, 174, 223

Belczynski, K., Repetto, S., Holz, D. E., et al. 2016c, ApJ, 819, 108

Belczynski, K., Sadowski, A., \& Rasio, F. A. 2004, ApJ, 611, 1068

Belczynski, K., Taam, R. E., Kalogera, V., Rasio, F. A., \& Bulik, T. 2007, ApJ, 662, 504

Belczynski, K., Wiktorowicz, G., Fryer, C. L., Holz, D. E., \& Kalogera, V. 2012, ApJ, 757, 91

Bennett, D. P., Becker, A. C., Quinn, J. L., et al. 2002, ApJ, 579, 639

Blaauw, A. 1961, BAN, 15, 265

Blagorodnova, N., Kotak, R., Polshaw, J., et al. 2017, ApJ, 834, 107

Bonnell, I. A., \& Pringle, J. E. 1995, MNRAS, 273, L12

Bray, J. C., \& Eldridge, J. J. 2018, MNRAS, 480, 5657

Breivik, K., Chatterjee, S., \& Larson, S. L. 2017, ApJL, 850, L13

Casares, J., Jonker, P. G., \& Israelian, G. 2017, Handbook of Supernovae (Berlin: Springer)

Chapline, G. F. 1975, Natur, 253, 251

Chruslinska, M., Nelemans, G., \& Belczynski, K. 2019, MNRAS, 482, 5012

Corral-Santana, J. M., Casares, J., Muñoz-Darias, T., et al. 2016, A\&A, 587, A61

de Mink, S. E., Sana, H., Langer, N., Izzard, R. G., \& Schneider, F. R. N. 2014, ApJ, 782, 7

Dominik, M., Belczynski, K., Fryer, C., et al. 2012, ApJ, 759, 52

Dominik, M., Belczynski, K., Fryer, C., et al. 2013, ApJ, 779, 72

Dominik, M., Berti, E., O'Shaughnessy, R., et al. 2015, ApJ, 806, 263

Dong, S., Mérand, A., Delplancke-Ströbele, F., et al. 2019, ApJ, 871, 70

Dong, S., Udalski, A., Gould, A., et al. 2007, ApJ, 664, 862

Duquennoy, A., \& Mayor, M. 1991, A\&A, 248, 485

Elbert, O. D., Bullock, J. S., \& Kaplinghat, M. 2018, MNRAS, 473, 1186

Eldridge, J. J., \& Stanway, E. R. 2016, MNRAS, 462, 3302

Farr, W. M., \& Mandel, I. 2018, Sci, 361, aat6506

Farr, W. M., Sravan, N., Cantrell, A., et al. 2011, ApJ, 741, 103

Ferrarese, L., \& Ford, H. 2005, SSRv, 116, 523

Fragos, T., Willems, B., Kalogera, V., et al. 2009, ApJ, 697, 1057
Fryer, C. L., Belczynski, K., Wiktorowicz, G., et al. 2012, ApJ, 749, 91

Fryer, C. L., \& Kalogera, V. 2001, ApJ, 554, 548

Fryer, C. L., \& Kusenko, A. 2006, ApJS, 163, 335

Giacobbo, N., \& Mapelli, M. 2018, MNRAS, 480, 2011

Glebbeek, E., Gaburov, E., Portegies Zwart, S., \& Pols, O. R. 2013, MNRAS, 434,3497

Gould, A. 2000, ApJ, 535, 928

Heger, A., \& Woosley, S. E. 2002, ApJ, 567, 532

Hobbs, G., Lorimer, D. R., Lyne, A. G., \& Kramer, M. 2005, MNRAS, 360, 974

Iben, I., Jr., \& Tutukov, A. V. 1997, ApJ, 491, 303

Ivanova, N., Justham, S., Chen, X., et al. 2013, A\&ARv, 21, 59

Ivanova, N., \& Taam, R. E. 2004, ApJ, 601, 1058

Janka, H.-T. 2013, MNRAS, 434, 1355

Jonker, P. G., \& Nelemans, G. 2004, MNRAS, 354, 355

Justham, S., Podsiadlowski, P., \& Vink, J. S. 2014, ApJ, 796, 121

Kaib, N. A., \& Raymond, S. N. 2014, ApJ, 782, 60

Kalogera, V., \& Baym, G. 1996, ApJL, 470, L61

Khlopov, M. Y. 2010, RAA, 10, 495

Klencki, J., Moe, M., Gladysz, W., et al. 2018, A\&A, 619, A77

Klencki, J., Wiktorowicz, G., Gładysz, W., \& Belczynski, K. 2017, MNRAS, 469, 3088

Kochanek, C. S., Adams, S. M., \& Belczynski, K. 2014, MNRAS, 443, 1319

Koliopanos, F. 2017, Proc. Multifrequency Behaviour High Energy Cosmic Sources Workshop 12 (Trieste: SISSA)

Kroupa, P. 2001, in ASP Conf. Ser. 228, Dynamics of Star Clusters and the Milky Way, ed. S. Deiters et al. (San Francisco, CA: ASP), 187

Kroupa, P., Tout, C. A., \& Gilmore, G. 1993, MNRAS, 262, 545

Kroupa, P., \& Weidner, C. 2003, ApJ, 598, 1076

Kruckow, M. U., Tauris, T. M., Langer, N., Kramer, M., \& Izzard, R. G. 2018, MNRAS, 481, 1908

Lamberts, A., Garrison-Kimmel, S., Hopkins, P., et al. 2018, MNRAS, 480,2704

Langer, N. 2012, ARA\&A, 50, 107

Leonard, P. J. T. 1989, AJ, 98, 217

Licquia, T. C., \& Newman, J. A. 2015, ApJ, 806, 96

Linden, T., Kalogera, V., Sepinsky, J. F., et al. 2010, ApJ, 725, 1984

Lipunov, V. M., Postnov, K. A., \& Prokhorov, M. E. 1997a, NA, 2, 43

Lipunov, V. M., Postnov, K. A., \& Prokhorov, M. E. 1997b, MNRAS, 288, 245

Lu, J. R., Lam, C. Y., Medford, M., Dawson, W., \& Golovich, N. 2019, RNAAS, 3, 58

Lyne, A. G., \& Lorimer, D. R. 1994, Natur, 369, 127

Maccarone, T. J., Chomiuk, L., Miller-Jones, J., et al. 2019, arXiv:1904.11842

Maeda, Y., Kubota, A., Kobayashi, Y., et al. 2005, ApJL, 631, L65

Mandel, I. 2016, MNRAS, 456, 578

Mandel, I., \& de Mink, S. E. 2016, MNRAS, 458, 2634

Mao, S., Smith, M. C., Woźniak, P., et al. 2002, MNRAS, 329, 349

Marchant, P., Renzo, M., Farmer, R., et al. 2019, ApJ, 882, 36

Mashian, N., \& Loeb, A. 2017, MNRAS, 470, 2611

Matsumoto, T., Teraki, Y., \& Ioka, K. 2018, MNRAS, 475, 1251

Mennekens, N., \& Vanbeveren, D. 2014, A\&A, 564, A134

Mezcua, M. 2017, IJMPD, 26, 1730021

Miller-Jones, J. C. A. 2014, PASA, 31, e016

Miyake, N., Udalski, A., Sumi, T., et al. 2012, ApJ, 752, 82

Motch, C., \& Pakull, M. W. 2012, MmSAI, 83, 415

Mróz, P., Udalski, A., Skowron, J., et al. 2017, Natur, 548, 183

Mroz, P., Udalski, A., Skowron, J., et al. 2019, ApJS, 244, 29

Nelemans, G., Yungelson, L. R., \& Portegies Zwart, S. F. 2001, A\&A, 375,890

Neugebauer, G. 2003, in The Collapse to a Black Hole, ed. H. Falcke \& F. W. Hehl (Bristol: Institute of Physics Publishing), 72

Nucita, A. A., De Paolis, F., Ingrosso, G., et al. 2006, ApJ, 651, 1092

Olejak, A., Belczynski, K., Bulik, T., \& Sobolewska, M. 2019, arXiv:1908. 08775

Osłowski, S., Moderski, R., Bulik, T., \& Belczynski, K. 2008, A\&A, 478, 429

Özel, F., Psaltis, D., Narayan, R., \& McClintock, J. E. 2010, ApJ, 725, 1918

Paczynski, B. 1986, ApJ, 304, 1

Paczynski, B. 1996, ARA\&A, 34, 419

Perna, R., Chruslinska, M., Corsi, A., \& Belczynski, K. 2018, MNRAS, 477, 4228

Peters, P. C. 1964, PhRv, 136, 1224

Podsiadlowski, P., Joss, P. C., \& Hsu, J. J. L. 1992, ApJ, 391, 246

Pooley, D., Kumar, P., Wheeler, J. C., \& Grossan, B. 2018, ApJL, 859, L23

Remillard, R. A., \& McClintock, J. E. 2006, ARA\&A, 44, 49

Renzo, M., Zapartas, E., de Mink, S. E., et al. 2019, A\&A, 624, A66

Repetto, S., Davies, M. B., \& Sigurdsson, S. 2012, MNRAS, 425, 2799 
Repetto, S., Igoshev, A. P., \& Nelemans, G. 2017, MNRAS, 467, 298

Repetto, S., \& Nelemans, G. 2015, MNRAS, 453, 3341

Robin, A. C., Reylé, C., Derrière, S., \& Picaud, S. 2003, A\&A, 409, 523

Rodriguez, C. L., Zevin, M., Pankow, C., Kalogera, V., \& Rasio, F. A. 2016, ApJL, 832, L2

Ruhlen, L., Smith, D. M., \& Swank, J. H. 2011, ApJ, 742, 75

Rybicki, K. A., Wyrzykowski, Ł., Klencki, J., et al. 2018, MNRAS, 476, 2013

Samland, M. 1998, ApJ, 496, 155

Sana, H. 2017, in IAU Symp. 329, The Lives and Death-Throes of Massive Stars, ed. J. J. Eldridge (Cambridge: Cambridge Univ. Press), 110

Sana, H., de Mink, S. E., de Koter, A., et al. 2012, Sci, 337, 444

Sana, H., Le Bouquin, J. B., Lacour, S., et al. 2014, ApJS, 215, 15

Schneider, F. R. N., Sana, H., Evans, C. J., et al. 2018, Sci, 359, 69

Shapiro, S. L., \& Teukolsky, S. A. 1983, Black Holes, White Dwarfs, and Neutron Stars: The Physics of Compact Objects (New York et al: Wiley)

Shvartsman, V. F. 1971, SvA, 15, 377

Shvartzvald, Y., Udalski, A., Gould, A., et al. 2015, ApJ, 814, 111

Singer, L. 2019, GCN, 24069

Skowron, J., Udalski, A., Gould, A., et al. 2011, ApJ, 738, 87

Spera, M., \& Mapelli, M. 2017, MNRAS, 470, 4739

Spera, M., Mapelli, M., Giacobbo, N., et al. 2019, MNRAS, 485, 889

Stevenson, S., Vigna-Gómez, A., Mandel, I., et al. 2017, NatCo, 8, 14906

Sumi, T., Bennett, D. P., Bond, I. A., et al. 2013, ApJ, 778, 150

Taam, R. E., \& Sandquist, E. L. 2000, ARA\&A, 38, 113

Tauris, T. M., \& van den Heuvel, E. P. J. 2006, Compact stellar X-ray sources (Cambridge: Cambridge Univ. Press), 623

The LIGO Scientific Collaboration, the Virgo Collaboration, Abbott, B. P., et al. 2019, PhRvX, 9, 031040

Thompson, T. A., Kochanek, C. S., Stanek, K. Z., et al. 2018, arXiv:1806.02751

Timmes, F. X., Woosley, S. E., \& Weaver, T. A. 1996, ApJ, 457, 834

Toonen, S., Hamers, A., \& Portegies Zwart, S. 2016, ComAC, 3, 6
Tout, C. A., Aarseth, S. J., Pols, O. R., \& Eggleton, P. P. 1997, MNRAS, 291, 732 Tsuna, D., \& Kawanaka, N. 2019, MNRAS, 488, 2099

Tsuna, D., Kawanaka, N., \& Totani, T. 2018, MNRAS, 477, 791

Tutukov, A. V., Yungelson, L. R., \& Iben, I. J. 1992, ApJ, 386, 197

Udalski, A., Szymański, M. K., \& Szymański, G. 2015, AcA, 65, 1

Venumadhav, T., Zackay, B., Roulet, J., Dai, L., \& Zaldarriaga, M. 2019, arXiv:1904.07214

Vigna-Gómez, A., Justham, S., Mandel, I., de Mink, S. E., \& Podsiadlowski, P. 2019, ApJL, 876, L29

Villante, F. L., Serenelli, A. M., Delahaye, F., \& Pinsonneault, M. H. 2014 ApJ, 787, 13

Vink, J. S. 2011, Ap\&SS, 336, 163

Vink, J. S. 2015, ASSL, 412, 77

Vink, J. S., Heger, A., Krumholz, M. R., et al. 2015, HiA, 16, 51

Voss, R., \& Tauris, T. M. 2003, MNRAS, 342, 1169

Webbink, R. F. 1984, ApJ, 277, 355

Wiktorowicz, G., Belczynski, K., \& Maccarone, T. 2014, in Binary Systems, their Evolution and Environments, 37

Williams, A. A., Belokurov, V., Casey, A. R., \& Evans, N. W. 2017, MNRAS, 468, 2359

Wongwathanarat, A., Janka, H.-T., \& Müller, E. 2013, A\&A, 552, A126

Woosley, S. E. 2016, ApJL, 824, L10

Woosley, S. E. 2017, ApJ, 836, 244

Woosley, S. E. 2019, ApJ, 878, 49

Woosley, S. E., Blinnikov, S., \& Heger, A. 2007, Natur, 450, 390

Wozniak, P. R., Udalski, A., Szymanski, M., et al. 2001, AcA, 51, 175

Wyrzykowski, Ł., Kostrzewa-Rutkowska, Z., Skowron, J., et al. 2016, MNRAS, 458, 3012

Wyrzykowski, Ł., \& Mandel, I. 2019, arXiv:1904.07789

Wyrzykowski, Ł., Rynkiewicz, A. E., Skowron, J., et al. 2015, ApJS, 216, 12 Yamaguchi, M. S., Kawanaka, N., Bulik, T., \& Piran, T. 2018, ApJ, 861, 21 